Pacific Journal of Mathematics

COHOMOLOGY OF COMODULES 


\title{
COHOMOLOGY OF COMODULES
}

\author{
Walter Ricardo Ferrer Santos
}

\begin{abstract}
The purpose of the present paper is to develop some aspects of a cohomology theory in the category of $C$ comodules, where $C$ is a bialgebra. This cohomology theory generalizes the rational cohomology of affine algebraic groups and also the cohomology of Lie Algebras. We develop the theory up to the point where we can guarantee the convergence of the analogue of the Hochschild Serre spectral sequence and the convergence of another spectral sequence, which is a natural generalization of the result known as Shapiro's lemma in the cohomology theory of finite groups.
\end{abstract}

1. Introduction. The attempts to generalize the Hochschild-Serre (or Lyndon-Hochschild-Serre) spectral sequence for the rational cohomology have a long history. One of the main difficulties was the fact that it is no longer evident (as it is for finite groups) that if $K$ is a closed normal subgroup of $G$ then the algebra $P(G)$ of polynomial functions on $G$ is injective as a $K$-module. A first remark about the need to prove that result was made by Hochschild in [4]. Recently, Cline, Parshall and Scott in [1] and Habousch in [3] proved the above injectivity result and established the validity of the spectral sequence. Here we prove a generalization of the above result, namely that if $C$ and $D$ are commutative Hopf Algebras over a field and $\pi: C \rightarrow D$ is a normal surjective Hopf Algebra map, then $C$ is injective as a $D$-comodule (Theorem 4.13).

We begin with a brief description of each section:

2. Cohomology of comodules. Here we introduce the cohomology theory with which we shall be concerned throughout the paper. We present it axiomatically and also construct an explicit resolution functor whose homology gives us the cohomology of comodules. The definition of this comodule cohomology seems to have been known to some specialists and was communicated to the author by G. Hochschild.

3. Normal maps and actions on the cohomology. This section is of a technical nature and has the main purpose of establishing the generalization to the category of comodules of the conjugation action of a group $G$ on the cohomology $H^{i}(K, M)$ where $M$ is a $G$-module and $K$ a normal subgroup of $G$. 
4. Induced comodules and injective comodules. Some of the constructions of this section generalize methods of [1] and [4]. We construct a functor $\pi^{*}$ that generalizes the induced representation functor, and we prove that $C$ is injective as $D$-comodule iff the functor $\pi^{*}$ is exact (Corollary 4.9). We use this criterion for showing that under certain normality assumptions $C$ is injective as a $D$-comodule (Theorem 4.13). Finally we prove Theorem 4.15 , which is a generalization of the condition for injectivity given in Proposition 2.2 of [5].

5. Two spectral sequences. We establish the validity of the Hochschild Serre spectral sequence for the cohomology of comodules as well as the spectral sequence of Theorem 5.3 that generalizes Shapiro's lemma on the cohomology of induced representations.

We shall follow the terminological conventions of [6]. Coalgebras, bialgebras and Hopf Algebras will be considered over a commutative ring $R$ with identity, or over an arbitrary field $k$. In the first case, we assume that they are flat when viewed as $R$-modules (we want the comodules to form an abelian category). If $C$ is a Hopf Algebra then $\Delta, \varepsilon$ will denote the comultiplication and counit, $\mu$ and $u$ will denote the multiplication and the unit, $\eta$ will stand for the antipode map, and $1_{C}$ will stand for the unit of $C$. If $M$ is a $C$-comodule then $\chi$ or $\chi_{M}$ will indicate the comodule structure on $M$, and we define $M^{C}=\left\{m \in \mid \chi_{M}(m)=m \otimes 1_{C}\right\}$. If $M$ and $N$ are $R$-modules then $M \otimes_{R} N$ will be written as $M \otimes N$ unless there is a danger of confusion. Given an $R$-coalgebra $C$ (that is flat as an $R$-module) the $C$-comodules, together with the $C$-comodule homomorphisms, form an abelian category that will be denoted by $\operatorname{CN}(C)$. The category of $R$-modules will be denoted by $\Re(R)$. If $M$ and $N$ are $R$ modules then $s: M \otimes N \rightarrow N \otimes M$ stands for the $R$-linear switching map sending each $m \otimes n$ onto $n \otimes m$. We use similar notation for any switching map, for example $s_{12,3}$ stands for the $R$-linear map sending each $x_{1} \otimes x_{2} \otimes x_{3}$ onto $x_{3} \otimes x_{1} \otimes x_{2}$.

Some of the results of this paper were part of the $\mathrm{PhD}$ dissertation of the author at U.C. Berkeley. That dissertation was written under the supervision of Professor G. Hochschild, and the author would like to thank him for his advice and help.

2. Cohomology of comodules. Let $C$ be a bialgebra over a ring $R$. An object $I$ in $\operatorname{Co}(C)$ is said to be relatively injective if it is injective with respect to the injective $C$-comodule maps that split when considered as $R$-module maps. The following two results are well known, we include them due to the lack of an adequate reference. 
Lemma 2.1. Let $X$ be an arbitrary $R$-module. Then the map id $\otimes \Delta$ is a $C$-comodule structure on $X \otimes C$. Moreover with this structure $X \otimes C$ is a relatively injective object in $\operatorname{Cg}(C)$.

Proof. Take a pair of objects $U$ and $V$ in $\operatorname{Cg}(C)$ and an injective $C$-comodule map $\alpha: U \rightarrow V$, for which there is an $R$-map $\beta: V \rightarrow U$ satisfying $\beta \alpha=\mathrm{id}_{U}$. Given any $C$-comodule map $t: U \rightarrow X \otimes C$ consider the map $\tilde{t}: \quad V \rightarrow X \otimes C$, given by $\tilde{t}=j(\mathrm{id} \otimes \varepsilon \otimes \mathrm{id})(t \otimes \mathrm{id})(\beta \otimes \mathrm{id}) \chi_{V}$, where $j$ is the map from $X \otimes R \otimes C$ to $X \otimes C$ defined by $j(x \otimes r \otimes c)=$ $r x \otimes c$. It is easy to check that $\tilde{t} \alpha=t$, and that $\tilde{t}$ is a $C$-comodule map.

LEMma 2.2. Every object in CগR $(C)$ can be relatively embedded in a relatively injective one.

Proof. Let $M$ be a $C$-comodule. Then $\chi_{M}: M \rightarrow M \otimes C$ is a $C$-comodule map when we endow $M$ with the structure $\chi_{M}$ and $M \otimes C$ with structure id $\otimes \Delta$. As $\chi_{M}$ is a relative embedding (follow it by id $\otimes \varepsilon$ ) our result follows from Lemma 2.1 .

Definition 2.3. We say that a $C$-comodule $Y$ is coinduced if it is of the form $X \otimes C$ with structure id ${ }_{X} \otimes \Delta$ for some $R$-module $X$.

If we consider the functor $M \rightarrow M^{C}$ from $\varrho \Re$ to $\Re(R)$ we see easily from the general theory of derived functors in abelian categories that the following result holds.

THEOREM 2.4. There is one, and (up to equivalence) only one cohomological functor $H^{n}(C,-), n \geq 0$, from $\mathrm{C} \Re(C)$ to $\Re(R)$ such that

(a) $H^{0}(C, M)=M^{0}$ for all $C$-comodules $M$.

(b) $H^{p}(C, Y)=0$ for every $p \geq 1$ and every coinduced comodule $Y$.

Now, we shall describe an explicit resolution functor and express the cohomology functors in terms of that resolution. Then we will describe them in terms of another more manageable resolution ("non homogeneous cochains").

Given any $R$-module $V$ consider $V \otimes C^{p}, p>0$, with structure $\mathrm{id}_{V \otimes C^{\mathrm{p}-1}} \otimes \Delta$ where $C^{p}$ stands for the $p$-fold tensor power of $C$, and $C^{0}=R$. If $V$ is a $C$-comodule we endow $V \otimes C^{0}=V$ with the structure $\chi_{V}$ and define the maps $d_{p}$ as follows:

(a) $d_{0}=\chi_{V}$,

(b) Given $d_{p-1}: V \otimes C^{p-1} \rightarrow V \otimes C^{p}$ define $d_{p}$ as

$$
d_{p}=d_{p-1} \otimes \mathrm{id}_{C}+(-1) \mathrm{id}_{V \otimes C^{p-1}} \otimes \Delta .
$$


It is not hard to prove inductively that

(1) $d_{p}$ is a C-comodule map for every $p \geq 0$.

(2) The sequence $\left(V \otimes C^{p}, d_{p}\right)$ is relatively exact, with splitting maps $\zeta_{p}: V \otimes C^{p} \rightarrow V \otimes C^{p-1}$ defined by $\zeta_{p}(x \otimes c)=(-1)^{p+1} \varepsilon(c) x$ with $x \in V \otimes C^{p-1}$ and $c \in C$. In order to verify (2) one has to check that $d_{p+1} d_{p}=0$ and that $\zeta_{1} d_{0}=\mathrm{id}$,

$$
\zeta_{p+1} d_{p}+d_{p-1} \zeta_{p}=\mathrm{id}_{V \otimes C^{p}} .
$$

We have

$$
\begin{aligned}
d_{p+1} d_{p}= & {\left[\left(d_{p} \otimes \mathrm{id}\right)+(-1)^{p+1} \mathrm{id}_{V \otimes C^{p}} \otimes \Delta\right] } \\
& \times\left[\left(d_{p-1} \otimes \mathrm{id}\right)+(-1)^{p} \mathrm{id}_{V \otimes C^{p-1}} \otimes \Delta\right] \\
= & (-1)^{p+1}\left(\mathrm{id}_{V \otimes C^{p}} \otimes \Delta\right)\left(d_{p-1} \otimes \mathrm{id}_{C}\right) \\
& +(-1)^{p}\left(d_{p} \otimes \mathrm{id}_{C}\right)\left(\mathrm{id}_{V \otimes C^{p-1}} \otimes \Delta\right) \\
& +(-1)^{2 p+1}\left(\mathrm{id}_{V \otimes C^{p}} \otimes \Delta\right)\left(\mathrm{id}_{V \otimes C^{p-1}} \otimes \Delta\right) .
\end{aligned}
$$

Applying the inductive definition of $d_{p}$ to the second summand above, we obtain

$$
\begin{aligned}
d_{p+1} d_{p}= & (-1)^{p+1}\left(\mathrm{id}_{V \otimes C^{p}} \otimes \Delta\right)\left(d_{p-1} \otimes \mathrm{id}_{C}\right) \\
& +(-1)^{p}\left(d_{p-1} \otimes \mathrm{id}_{C} \otimes \mathrm{id}_{C}+(-1)^{p} \mathrm{id}_{V \otimes C^{p-1}} \otimes \Delta \otimes \mathrm{id}_{C}\right) \\
& \times\left(\mathrm{id}_{V \otimes C^{p-1}} \otimes \Delta\right) \\
& +(-1)^{2 p+1}\left(\mathrm{id}_{V \otimes C^{p}} \otimes \Delta\right)\left(\mathrm{id}_{V \otimes C^{p-1}} \otimes \Delta\right),
\end{aligned}
$$

and the terms cancel in pairs. The other statements in (2) can be proven in a similar fashion.

Let us call $e(V)$ the complex

$$
\mathrm{e}(V): V \stackrel{d_{0}}{\rightarrow} V \otimes C \stackrel{d_{1}}{\rightarrow} \cdots \rightarrow V \otimes C^{p-1} \stackrel{d_{p, 1}}{\rightarrow} V \otimes C^{p} \stackrel{d_{p}}{\rightarrow} \cdots .
$$

This is a relatively injective resolution of $V$. Consequently, $H^{n}(C, V)$ may be identified with the homology of the complex $\mathscr{D}(V)$ obtained applying the first part functor to $C(V)$ and deleting the first $R$-module.

$$
\begin{aligned}
\mathscr{D}(V): 0 & \rightarrow(V \otimes C)^{C} \stackrel{d_{1}}{\rightarrow}\left(V \otimes C^{2}\right)^{C} \rightarrow \\
& \rightarrow \cdots \rightarrow\left(V \otimes C^{p-1}\right)^{C} \stackrel{d_{p-1}}{\rightarrow}\left(V \otimes C^{p}\right)^{C} \rightarrow \cdots .
\end{aligned}
$$


If we define $\gamma_{p-1}: V \otimes C^{p-1} \rightarrow V \otimes C^{p}$ by $\gamma_{p-1}(x)=x \otimes 1_{C}$, then $\gamma_{p-1}$ is a bijective $R$-map between $V \otimes C^{p-1}$ and $\left(V \otimes C^{p}\right)^{C}$. A direct computation shows that the differential $d_{p}$ when restricted to $\left(V \otimes C^{p}\right)^{C}$ induces a map $\delta_{p}=\gamma_{p}^{-1} d_{p} \gamma_{p-1}: V \otimes C^{p-1} \rightarrow V \otimes C^{p}$, given by

$$
\begin{array}{r}
\delta_{1}=d_{0}-\gamma_{0} \quad \text { or } \quad \delta_{1}(x)=\chi_{V}(x)-x \otimes 1_{C}, \quad \text { and } \\
\delta_{p+1}=\delta_{p} \otimes \mathrm{id}_{C}+(-1)^{p+1}\left(\mathrm{id}_{V \otimes C^{p}} \otimes 1_{C}-\mathrm{id}_{V \otimes C^{p-1}} \otimes \Delta\right. \\
\left.+\mathrm{id}_{V \otimes C^{p-1}} \otimes 1_{C} \otimes \mathrm{id}_{C}\right) .
\end{array}
$$

This shows that the complex $\mathscr{D}(V)$ is isomorphic to the complex

$$
\mathcal{E}(V): 0 \rightarrow V \stackrel{\delta_{1}}{\rightarrow} V \otimes C \stackrel{\delta_{2}}{\rightarrow} \cdots \rightarrow V \otimes C^{p-1} \stackrel{\delta_{n}}{\rightarrow} V \otimes C^{p} \stackrel{\delta_{p+1}}{\rightarrow} \cdots
$$

Thus, we can compute $H^{n}(C, V)$ explicitly as the homology of the complex above.

Let us write $\delta_{1}$ and $\delta_{2}$ explicitly

$$
\begin{aligned}
\delta_{1}(x) & =\chi_{V}(x)-x \otimes 1_{C} . \\
\delta_{2}(x) & =\delta_{1} \otimes \mathrm{id}_{C}+\left(\mathrm{id}_{V \otimes C} \otimes 1_{C}-\mathrm{id} \otimes \Delta+\mathrm{id}_{V} \otimes 1_{C} \otimes \mathrm{id}_{C}\right) \\
& =\chi_{V} \otimes \mathrm{id}_{C}+\mathrm{id}_{V \otimes C} \otimes 1_{C}-\mathrm{id} \otimes \Delta .
\end{aligned}
$$

In other words

$$
\delta_{2}(v \otimes c)=\chi(v) \otimes c+v \otimes c \otimes 1_{C}-v \otimes \Delta(c) .
$$

Hence, we have

$$
\begin{aligned}
& H^{1}(C, V) \\
& \quad=\left\{\sum v_{l} \otimes c_{l} \mid \sum_{l} \chi_{V}\left(v_{l}\right) \otimes c_{l}=-\sum_{i} v_{i} \otimes c_{l} \otimes 1_{C}+\sum_{i} v_{l} \otimes \Delta\left(c_{l}\right)\right\} / U,
\end{aligned}
$$

where $U=\left\{\chi_{V}(x)-x \otimes 1_{C} \mid x \in V\right\}$. In the case where the comodule structure of $V$ is trivial we have

$$
H^{1}(C, V)=V \otimes C_{0} \quad \text { where } C_{0}=\{x \in C \mid \Delta(x)=x \otimes 1+1 \otimes x\} .
$$

Recall that the trivial comodule structure on $V$ is the one that maps $v$ into $v \otimes 1$, for every $v$ in $V$.

3. Normal maps and actions on the cohomology. Let $G$ be a finite group and $K$ a normal subgroup. For every $G$-module $M$ there is an action of $G$ on $H^{\prime}(K, M)$ having the following properties: 
(a) For every exact sequence $0 \rightarrow M \rightarrow N \rightarrow S \rightarrow 0$ of $G$-modules, all the morphisms of the long exact $K$-cohomology sequence

$$
\cdots \rightarrow H^{\prime}(K, S) \rightarrow H^{t+1}(K, M) \rightarrow H^{\prime+1}(K, N) \rightarrow H^{i+1}(K, S) \rightarrow \cdots
$$

are $G$-maps.

(b) The action of $G$ on $H^{0}(K, M)$ coincides with the one induced on $M^{K}$ by the action of $G$ on $M$.

(c) $K$ acts trivially on $H^{\prime}(K, M)$ for every $i$.

The natural generalization of this situation would be to take a pair of Hopf Algebras $C$ and $D$ and a normal surjective bialgebra map $\pi: C \rightarrow D$ and construct a $C^{D}$-comodule structure on $H^{\prime}(D, M)$ for every $C$-comodule $M$. In order to shorten certain long computations and have a simpler notation we shall consider the following (nominally) more general situation.

Definition 3.1. Let $C$ and $D$ be Hopf Algebras over a commutative ring $R$ (as usual these are supposed to be flat as $R$ modules).An adjoint $C$-structure on $D$ is a $C$-comodule structure $\theta$ on $D$ such that the following diagrams are commutative.
(1) $D \stackrel{\theta}{\rightarrow} D \otimes C$
$D \stackrel{\theta}{\rightarrow} D \otimes C$
$\uparrow u \quad \uparrow u \otimes u$
$\downarrow \Delta \quad \downarrow \Delta \otimes$ id
$R \rightarrow R \otimes R$
(3) $\quad \stackrel{\theta}{\rightarrow} D \otimes C$
$\downarrow \varepsilon \quad \downarrow \varepsilon_{D} \otimes \varepsilon_{C}$,
$R \rightarrow R \otimes R$

where $\theta \otimes \theta$ is the tensor product comodule structure whose properties we recall below.

For any bialgebra $C$ and for any pair of $C$-comodules $M$ and $N$ with structures $\chi_{M}$ and $\chi_{N}$, the map

$$
\chi_{M} \otimes \chi_{N}=(\mathrm{id} \otimes \mathrm{id} \otimes \mu)\left(\mathrm{id} \otimes{ }_{S} \otimes \mathrm{id}\right)\left(\chi_{M} \otimes \chi_{N}\right)
$$

is a $C$ comodule structure on $M \otimes N$. Recall that $s$ stands for the switching map. When we consider the $R$-module $M \otimes N$ with the structure $\chi_{M} \otimes \chi_{N}$ we shall denote it as $M \otimes N$. It is easy to prove that if $f: M \rightarrow M^{\prime}, g: N \rightarrow N^{\prime}$ are $C$-comodule maps, then

$$
f \otimes g: M \otimes N \rightarrow M^{\prime} \otimes N^{\prime}
$$

is a $C$-comodule map.

Definition 3.2. Let $\alpha$ be a covariant functor from CoR $(C)$ to Cok $(D)$ having the following properties:

(a) For every object $\left(M, \chi_{M}\right)$ in $\operatorname{Cg}(C), \alpha\left(M, \chi_{M}\right)=\left(M, \chi_{M \alpha}\right)$ for some $D$-comodule structure $\chi_{M \alpha}$ on $M$. 
(b) For any $f \in \operatorname{Hom}_{C}(M, N) \alpha(f)=f$.

(c) $\alpha(M \otimes N)=\alpha(M) \otimes \alpha(N)$ (i.e., $\left(\chi_{M} \otimes \chi_{N}\right)_{\alpha}=\chi_{M \alpha} \otimes \chi_{N \alpha}$ ).

(d) If $u_{C}: R \rightarrow R \otimes C$ is the unit of $C$, viewed as a $C$-comodule structure on $R$, then $u_{C \alpha}: R \rightarrow R \otimes D$ coincides with the unit of $D$.

We say the $\alpha$ is compatible with the adjoint structure $\theta$ if, moreover, $\alpha$ satisfies the following condition:

(e) For every object $\left(M, \chi_{M}\right)$ of $\operatorname{eN}(C), \chi_{M \alpha}: M \rightarrow M \otimes D$ is a $C$-comodule map from $\left(M, \chi_{M}\right)$ to $\left(M \otimes D, \chi_{M} \otimes \theta\right)$.

LEMMA 3.3. In the situation above, the following four conditions are satisfied:

(a) If $M$ is a $C$-comodule and $f \in \operatorname{Hom}_{R}(M, R)$, the maps $\Delta$ and $(\mathrm{id} \otimes \mu)(s \otimes \mathrm{id})\left(\mathrm{id} \otimes \theta_{\alpha}\right) \Delta$ coincide on every $R$-submodule of $D$ of the form $\tilde{f}(M)$ where $\tilde{f}: M \rightarrow D$ is given by $\tilde{f}(x)=\Sigma_{i} f\left(x_{\imath}\right) d$, where $\chi_{M \alpha}(x)=$ $\sum x_{1} \otimes d_{1}$. (More briefly: $\tilde{f}=\left(f \otimes \mathrm{id}_{D}\right) \chi_{M \alpha}$.)

(b) If $M$ is a $C$-comodule with structure $\chi_{M}$, then the following diagram is commutative:

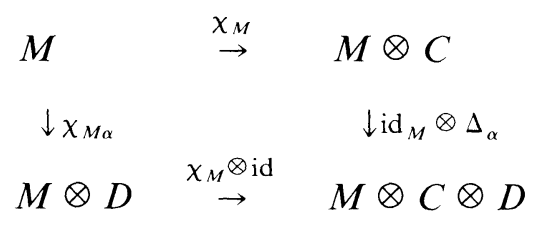

where $\Delta_{\alpha}$ stands for $\Delta_{C \alpha}: C \rightarrow C \otimes D$.

(c) $\Delta_{\alpha}\left(1_{C}\right)=1_{C} \otimes 1_{D}, \theta_{\alpha}\left(1_{D}\right)=1_{D} \otimes 1_{D}$.

(d) $\theta_{\alpha}$ is an adjoint $D$-structure on $D$.

Proof. (a) Condition (e) of Definition 3.2 means that $\left(\chi_{M} \otimes \theta\right) \chi_{M \alpha}=$ $\left(\chi_{M \alpha} \otimes \mathrm{id}\right) \chi_{M}$. Conditions (b) of Definition 3.2, applied to $f=\chi_{M \alpha}$, allows us to deduce that $\left(\chi_{M} \otimes \theta\right)_{\alpha} \chi_{M \alpha}=\left(\chi_{M \alpha} \otimes\right.$ id $) \chi_{M \alpha}$. After applying condition (c) of Definition 3.2 and the definition of $\otimes$, we have

$(\mathrm{id} \otimes \mathrm{id} \otimes \mu)(\mathrm{id} \otimes s \otimes \mathrm{id})\left(\mathrm{id} \otimes \mathrm{id} \otimes \theta_{\alpha}\right)\left(\chi_{M \alpha} \otimes \mathrm{id}\right) \chi_{M \alpha}=$

$$
=\left(\chi_{M \alpha} \otimes \mathrm{id}\right) \chi_{M \alpha},
$$

from which we deduce

$(\mathrm{id} \otimes \mathrm{id} \otimes \mu)(\mathrm{id} \otimes s \otimes \mathrm{id})\left(\mathrm{id} \otimes \mathrm{id} \otimes \theta_{\alpha}\right)(\mathrm{id} \otimes \Delta) \chi_{M \alpha}=(\mathrm{id} \otimes \Delta) \chi_{M \alpha}$. If $\chi_{M \alpha}(m)=\sum m_{l} \otimes d_{l}$, we have

$$
\sum m_{l} \otimes(\mathrm{id} \otimes \mu)(s \otimes \mathrm{id})\left(\mathrm{id} \otimes \theta_{\alpha}\right) \Delta\left(d_{l}\right)=\sum m_{l} \otimes \Delta\left(d_{l}\right) .
$$

Applying $f$ we deduce our result. 
(b) Next we see immediately from part (b) of Definition 3.2, applied to the map $\chi_{M}: M \rightarrow M \otimes C$, that the diagram

$$
\begin{array}{lcc}
M & \stackrel{\chi_{M}}{\rightarrow} & M \otimes C \\
\downarrow \chi_{M \alpha} & & \downarrow\left(\mathrm{id}_{M} \otimes \Delta\right)_{\alpha} \\
M \otimes D & \stackrel{\chi_{M} \otimes \text { id }}{\rightarrow} & M \otimes C \otimes D
\end{array}
$$

is commutative. Now, if we endow $M$ with the trivial $D$-comodule structure $\chi_{0}: M \rightarrow M \otimes D$ given by $\chi_{0}(m)=m \otimes 1_{D}$, we have $\chi_{0} \otimes \Delta=$ $\mathrm{id}_{M} \otimes \Delta$, because

$$
\begin{aligned}
& (\mathrm{id} \otimes \mathrm{id} \otimes \mu)(\mathrm{id} \otimes s \otimes \mathrm{id})(\mathrm{id} \otimes \mathrm{id} \otimes \Delta)\left(\chi_{0} \otimes \mathrm{id}\right)(m \otimes d) \\
& \quad=(\mathrm{id} \otimes \mathrm{id} \otimes \mu)(\mathrm{id} \otimes s \otimes \mathrm{id})(m \otimes 1 \otimes \Delta(d))=m \otimes \Delta(d) .
\end{aligned}
$$

Therefore, $\left(\mathrm{id}_{M} \otimes \Delta\right)_{\alpha}=\left(\chi_{0} \otimes \Delta\right)_{\alpha}=\chi_{0 \alpha} \otimes \Delta_{\alpha}=\mathrm{id}_{M} \otimes \Delta_{\alpha}$, so that condition (b) is established. Finally, (c) and (d) follow immediately from the definitions.

Let $M$ be an arbitrary $C$-comodule and consider the complex of $R$-modules, $\mathcal{E}(M)$ defined as in $\S 2$ as follows:

$$
\begin{aligned}
& \mathscr{E}(M): M \stackrel{\delta_{1}}{\rightarrow} M \otimes D \stackrel{\delta_{2}}{\rightarrow} M \otimes D^{2} \rightarrow \cdots \rightarrow M \otimes D^{p-1} \stackrel{\delta_{p}}{\rightarrow} M \otimes D^{p} \rightarrow \cdots, \\
& \delta_{1}=\chi_{M \alpha}-\mathrm{id}_{M} \otimes 1_{D}, \\
& \quad \delta_{p+1}=\delta_{p} \otimes \mathrm{id}_{D}+\quad \\
& \quad+(-1)^{p}\left(\mathrm{id}_{M \otimes D^{p+1}} \otimes \Delta-\mathrm{id}_{M \otimes D^{p}} \otimes 1_{D}-\mathrm{id}_{M \otimes D^{p}} \otimes 1_{D} \otimes \mathrm{id}\right) .
\end{aligned}
$$

We proved in $\S 2$ that the homology of this complex is $H^{\prime}(D, \alpha(M))$. Now consider the maps $\chi_{k}: M \otimes D^{k} \rightarrow M \otimes D^{k} \otimes C$ defined inductively as follows, $\chi_{0}=\chi_{M}, \chi_{k}=\chi_{k-1} \otimes \theta$. Using the properties of $\otimes$ mentioned after Definition 3.1 and induction we see that $\chi_{k}$ is a $C$-comodule structure on $M \otimes D^{k}$.

Lemma 3.4. The maps $\delta_{h}$ are C-comodule maps when $M \otimes D^{h}$ is endowed with the structure $\chi_{h}$.

Proof. The proof will proceed by induction and will use repeatedly the properties of $\otimes$ stated after Definition 3.1. We want to verify that

$$
\chi_{p+1} \delta_{p+1}=\left(\delta_{p+1} \otimes \mathrm{id}_{C}\right) \chi_{p} .
$$


For $p=0$, this says that

$$
\left(\chi_{M} \otimes \theta\right)\left(\chi_{M \alpha}-\mathrm{id}_{M} \otimes 1_{D}\right)=\left(\chi_{M \alpha} \otimes \mathrm{id}_{C}-\mathrm{id}_{M} \otimes 1_{D} \otimes \mathrm{id}_{C}\right) \chi_{M}
$$

and it suffices to prove that

$$
\left(\chi_{M} \otimes \theta\right) \chi_{M \alpha}=\left(\chi_{M \alpha} \otimes \mathrm{id}_{C}\right) \chi_{M}
$$

and

$$
\left(\chi_{M} \otimes \theta\right)\left(\mathrm{id}_{M} \otimes 1_{D}\right)=\left(\mathrm{id}_{M} \otimes 1_{D} \otimes \mathrm{id}_{C}\right) \chi_{M} .
$$

The first equality is nothing but condition (e) of Definition 3.2, the second follows easily from the fact that $\theta\left(1_{D}\right)=1_{D} \otimes 1_{C}$. As to the general case, $\delta_{p+1}$ is defined as the sum of four maps (see definition above), and we shall verify that each of the four summands in a $C$-comodule map. For the first summand, we have to verify that

$$
\left(\chi_{p} \otimes \theta\right)\left(\delta_{p} \otimes \mathrm{id}_{C}\right)=\left(\delta_{p} \otimes \mathrm{id} \otimes \mathrm{id}_{C}\right)\left(\chi_{p-1} \otimes \theta\right) .
$$

By induction we have

$$
\chi_{p} \delta_{p}=\left(\delta_{p} \otimes \mathrm{id}_{C}\right) \chi_{p-1} .
$$

Using that $\theta \mathrm{id}_{C}=\left(\mathrm{id}_{C} \otimes \mathrm{id}_{D}\right) \theta$, we deduce after taking tensor products (see comments after Def. 3.1), that

$$
\left(\chi_{p} \otimes \theta\right)\left(\delta_{p} \otimes \mathrm{id}_{C}\right)=\left(\delta_{p} \otimes \mathrm{id}_{C} \otimes \mathrm{id}_{D}\right)\left(\chi_{p-1} \otimes \theta\right) .
$$

For the other summands of $\delta_{p+1}$, the proof is based on the same principles.

Using a similar inductive argument we prove

Lemma 3.5. Let $f: M \rightarrow N$ be a C-comodule map. Then the maps $f \otimes \mathrm{id}_{D^{p}}: M \otimes D^{p} \rightarrow N \otimes D^{p}$ are C-comodule maps when we endow $M \otimes D^{p}$ and $N \otimes D^{p}$ with the structures $\chi_{p}$.

Theorem 3.6. Let $C, D, \theta, \alpha$ be as before. Then, for every $C$-module $M$, there is a $C$-comodule structure $\tilde{\chi}_{k}$ on $H^{k}(D, \alpha(M))$ such that

(a) $\tilde{\chi}_{0}$ coincides with $\chi_{M}$.

(b) For every exact sequence of $C$-comodules $0 \rightarrow M \rightarrow N \rightarrow S \rightarrow 0$, all the maps in the long exact sequence

$$
\begin{aligned}
\cdots & \rightarrow H^{i}(D, \alpha(M)) \rightarrow H^{\prime}(D, \alpha(N)) \\
& \rightarrow H^{\prime}(D, \alpha(S)) \rightarrow H^{i+1}(D, \alpha(M)) \rightarrow \cdots
\end{aligned}
$$

are C-comodule maps. 
Proof. The existence of $\tilde{\chi}_{k}$ follows from Lemma 3.4. The fact that $\tilde{\chi}_{0}$ coincides with $\chi_{M}$ follows from the very definition of $\chi_{0}$. The fact that the maps $H^{\prime}(D, \alpha(M)) \rightarrow H^{\prime}(D, \alpha(N))$ and $H^{\prime}(D, \alpha(N)) \rightarrow H^{\prime}(D, \alpha(S))$ are $C$-comodule maps follow from Lemma 3.5. The only part that still requires proof is the assertion that the connecting homomorphisms are $C$-comodule maps. This will follow from the considerations below.

Let $X$ be an arbitrary bialgebra that is flat when considered as an $R$-module, and let $\mathbf{A}$ be a complex of $X$-comodules and $X$-comodule maps. We define an $X$-comodule structure on $H(\mathbf{A})$ as follows. Let $r+d_{t-1}\left(A_{l-2}\right) \in H^{t-1}(\mathbf{A})$ then $(d \otimes \mathrm{id}) \chi(r)=\chi d r=\chi 0=0$, since $X$ is $R$-flat this shows that $\chi(r) \in(\operatorname{Ker} d) \otimes X$, i.e., there are $r_{j} \in \operatorname{Ker} d$ and $x, \in X$ such that $\chi(r)=\sum r_{1} \otimes x_{1}$. If $r \in d_{1-1}\left(A_{t-2}\right)$ we have $r=d r_{1}$ and then

$$
\chi(r)=\chi\left(d r_{1}\right)=(d \otimes \mathrm{id}) \chi\left(r_{1}\right) \in d_{t-1}\left(A_{l-2}\right) \otimes X .
$$

Thus $\chi$ induces a map $\tilde{\chi}$ from $H(\mathbf{A})$ to $H(\mathbf{A}) \otimes X$ where

$$
\tilde{\chi}\left(r+d_{l-1}\left(A_{\imath-2}\right)\right)=\sum_{j}\left(r_{j}+d_{i-1}\left(A_{l-2}\right)\right) \otimes x_{j} .
$$

Let $0 \rightarrow \mathbf{A} \rightarrow \mathbf{B} \rightarrow \mathbf{C} \rightarrow 0$ be a short exact sequence in the category of

\begin{tabular}{|c|c|c|c|c|c|c|c|}
\hline \multirow{2}{*}{0} & $\rightarrow$ & $A_{l-2}$ & $\stackrel{\alpha_{t-2}}{\rightarrow}$ & $B_{t-2}$ & $\stackrel{\beta_{t-2}}{\rightarrow}$ & $C_{i-2}$ & $\rightarrow$ \\
\hline & & $\downarrow d_{1,1}$ & & $\downarrow d_{1,1}$ & & $\downarrow d_{1,1}$ & \\
\hline 0 & $\rightarrow$ & $A_{\imath-1}$ & $\stackrel{\alpha_{i-1}}{\rightarrow}$ & $B_{t-1}$ & $\stackrel{\beta_{l-1}}{\rightarrow}$ & $C_{l-1}$ & $\rightarrow$ \\
\hline & & $\downarrow d_{i}$ & & $\downarrow d_{t}$ & & $\downarrow d$, & \\
\hline 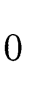 & $\rightarrow$ & $A_{\imath}$ & $\stackrel{\alpha_{i}}{\rightarrow}$ & $B_{t}$ & $\stackrel{\beta_{l}}{\rightarrow}$ & $C_{l}$ & $\rightarrow$ \\
\hline & & $\downarrow d_{1+1}$ & & $\downarrow d_{1+1}$ & & $\downarrow d_{t+1}$ & \\
\hline & $\rightarrow$ & $A_{\imath+1}$ & $\stackrel{\alpha_{t+1}}{\rightarrow}$ & $B_{t+1}$ & $\stackrel{\beta_{i+1}}{\rightarrow}$ & $C_{l+1}$ & $\rightarrow$ \\
\hline
\end{tabular}
complexes of $X$-comodules. We have the diagram

where all the squares are commutative, all the rows are exact, all the objects are $X$-comodules and all the maps are $X$-comodule maps. Now we recall the definition of $\delta: H^{t^{-1}}(\mathbf{C}) \rightarrow H^{\prime}(\mathbf{A})$. Take an element $c \in C_{l-1}$ such that $d_{l} c=0$ and consider $a \in A_{l}$ such that $\alpha_{i} a=d_{i} b$ with $b \in B_{l-1}$ such that $\beta_{i-1} b=c$. Then, as $\alpha_{1+1} d_{t+1} a=d_{i+1} \alpha_{i} a=d_{1+1} d_{l} b=0$, we have $a \in \operatorname{Ker} d_{t+1}$. In these terms we have $\delta\left(c+d_{t-1}\left(C_{t-2}\right)\right)=$ $a+d_{l-1}\left(A_{l-1}\right)$. Let $\chi(a)=\sum a_{k} \otimes x_{k}$ with $a_{k} \in \operatorname{Ker} d_{l+1}$. Then $\chi \alpha a=$ $(\alpha \otimes \mathrm{id}) \chi a=\sum a_{k} \otimes x_{k}$. Thus $\chi d b=\sum \alpha a_{k} \otimes x_{k}$. On the other hand, if 
$\chi b=\Sigma b_{j} \otimes y_{j}$, then $\chi d b=\Sigma d b_{j} \otimes y_{j}$. Thus, we have the equality

$$
\sum d b_{j} \otimes y_{j}=\sum \alpha a_{k} \otimes x_{k} .
$$

Now $\chi(c)=\chi \beta b=(\beta \otimes \mathrm{id}) \chi b=\sum \beta b_{j} \otimes y_{j}$. Now take $t_{r} \in \operatorname{Ker} d$ and $z_{r} \in X$ such that $\chi(c)=\sum t_{r} \otimes z_{r}$. Then

$$
\tilde{\chi}\left(c+d_{i-1}\left(C_{i-2}\right)\right)=\sum_{r}\left(t_{r}+d_{i-1}\left(C_{i-2}\right)\right) \otimes z_{r} .
$$

Consequently, we have

$$
(\delta \otimes \mathrm{id}) \tilde{\chi}\left(c+d_{i-1}\left(C_{i-2}\right)\right)=\sum \delta\left(t_{r}+d_{i-1}\left(C_{i-2}\right)\right) \otimes z_{r} .
$$

Take $m_{r} \in B_{i-1}$ such that $\beta m_{r}=t_{r}$ and then choose $s_{r} \in A_{i}$ such that $d m_{r}=\alpha s_{r}$. Now, we have

$$
(\delta \otimes \mathrm{id}) \tilde{\chi}\left(c+d_{i-1}\left(C_{i-2}\right)\right)=\sum\left(s_{r}+d_{i}\left(A_{i-1}\right)\right) \otimes z_{r} .
$$

On the other hand,

$$
\tilde{\chi}\left(\delta\left(c+d_{i-1}\left(C_{i-2}\right)\right)\right)=\tilde{\chi}\left(a+d_{i}\left(A_{i-1}\right)\right)=\sum\left(a_{k}+d_{i}\left(A_{i-1}\right)\right) \otimes x_{k} .
$$

From $\Sigma \beta b_{j} \otimes y_{j}=\chi(c)=\Sigma t_{r} \otimes z_{r}$, we deduce that

$$
\sum \beta b_{j} \otimes y_{j}=\sum \beta m_{r} \otimes z_{r} \text {. }
$$

Consequently,

$$
\sum b_{J} \otimes y_{j}-\sum m_{r} \otimes z_{r} \in \operatorname{Ker}(\beta \otimes \mathrm{id})=\operatorname{Ker}(\beta) \otimes X=\operatorname{Im} \alpha \otimes X .
$$

Thus, there are elements $a_{l}^{\prime}$ in $A_{i-1}$ and $w_{l}$ in $X$ such that

$$
\sum b_{j} \otimes y_{j}-\sum m_{r} \otimes z_{r}=\sum \alpha\left(a_{l}^{\prime}\right) \otimes w_{l} \text {. }
$$

Hence, $\Sigma d b_{j} \otimes y_{j}-\sum d m_{r} \otimes z_{r}=\sum d \alpha a_{l}^{\prime} \otimes w_{l}$. Using $d m_{r}=\alpha s_{r}$ and (1) we obtain

$$
\sum \alpha a_{k} \otimes x_{k}-\sum \alpha s_{r} \otimes z_{r}=\sum \alpha d a_{l}^{\prime} \otimes w_{l} .
$$

As $\alpha$ is injective and $X$ is flat as an $R$-module, we conclude that

$$
\sum a_{k} \otimes x_{k}-\sum s_{r} \otimes z_{r}=\sum d a_{l}^{\prime} \otimes w_{l} .
$$

Then

$$
\sum\left(s_{r}+d_{i}\left(A_{i-1}\right)\right) \otimes z_{r}=\sum\left(a_{k}+d_{i}\left(A_{t-1}\right)\right) \otimes x_{k},
$$

i.e., $\tilde{\chi} \delta=(\delta \otimes \mathrm{id}) \tilde{\chi}$.

The following results generalize the fact that for finite groups not only $G$ acts on the cohomology $H^{i}(K, M)$ by conjugation, but that this action factors through $K$, giving an action of $G / K$ on $H^{i}(K, M)$. 
THEOREM 3.7. Let $X$ be a Hopf Algebra over a commutative ring $R$ and let $\theta_{X}$ be an adjoint $X$-structure on $X$. Then if $(\mathrm{id} \otimes \mu)(s \otimes \mathrm{id})\left(\mathrm{id} \otimes \theta_{X}\right) \Delta=$ $\Delta$, the identity functor id: C目 $(X) \rightarrow \operatorname{Cg}(X)$ is compatible with the structure $\theta_{X}$. Moreover, the structure $\tilde{\chi}_{k}$ defined as in Theorem 3.6 is trivial (i.e. $\tilde{\chi}_{k}(x)=x \otimes 1$ for every $x \in H^{k}(X, M)$ ).

Proof. Referring to Definition 3.2, conditions (a), (b), (c) and (d) are trivially verified. As to (e), we have to prove that if $\chi_{M}$ is an $X$-comodule structure on $M$, then

$(\mathrm{id} \otimes \mathrm{id} \otimes \mu)(\mathrm{id} \otimes s \otimes \mathrm{id})\left(\mathrm{id} \otimes \mathrm{id} \otimes \theta_{X}\right)(\chi \otimes \mathrm{id}) \chi=(\chi \otimes \mathrm{id}) \chi$

It is clear that $\left(\chi_{M} \otimes \mathrm{id}\right) \chi_{M}=(\mathrm{id} \otimes \Delta) \chi_{M}$ and our hypothesis, imply the equality above. As to the triviality of $\tilde{\chi}_{k}$ we proceed by induction (dimension shifting). The axioms of the cohomology theory we are looking at, guarantee, for every $M$, the existence of an exact sequence $0 \rightarrow M \rightarrow$ $X_{M} \rightarrow S_{M} \rightarrow 0$ in the category $\operatorname{Cr}(X)$ such that the maps

$$
\delta: H^{\prime}\left(X, S_{M}\right) \rightarrow H^{i+1}(X, M)
$$

are surjective for every $i \geq 0$. By Theorem 3.6, $\tilde{\chi}_{1+1} \delta=$ $(\delta \otimes \mathrm{id}) \tilde{\chi}_{1}$. Given $x \in H^{\prime+1}(X, M)$ there is a $y \in H^{\prime}\left(X, S_{M}\right)$ such that $\delta y=x$. Then, $\tilde{\chi}_{l+1}(x)=\tilde{\chi}_{l+1} \delta y=(\delta \otimes$ id $) \tilde{\chi}_{l}(y)$. If $\tilde{\chi}_{l}(y)=y \otimes 1$ we deduce that $\tilde{\chi}_{t+1}(x)=x \otimes 1$. As the result is obvious for $k=0$, our proof is finished.

Now, let us return to the situation of Theorem 3.6. Let $\Delta$ be the comultiplication on $C$, and let $\Delta_{\alpha}: C \rightarrow C \otimes D$ be the $D$-structure associated with $\Delta$ via $\alpha$. Assume that the map $U: C \rightarrow D$ given by $U(x)=$ $\sum \varepsilon\left(c_{l}\right) d_{l}$ with $\Delta_{\alpha}(x)=\sum x_{l} \otimes d_{l}$, is surjective. Lemma 3.3 part (a) guarantees that $\theta_{\alpha}$ verifies (id $\left.\otimes \mu\right)(s \otimes \mathrm{id})\left(\mathrm{id} \otimes \theta_{\alpha}\right) \Delta_{D}=\Delta_{D}$. Consequently, by Theorem 3.7, $\theta_{\alpha}$ will be an adjoint $D$-structure on $D$ compatible with the identity functor on $\operatorname{CN}(D)$. Now consider the map $\chi_{k}-\chi_{M} \otimes \theta \otimes \ldots \otimes \theta$ from $M \otimes D^{k}$ to $M \otimes D^{k} \otimes C$. We have $\chi_{k \alpha}=$ $\chi_{M \alpha} \otimes \theta_{\alpha} \otimes \cdots \otimes \theta_{\alpha}$. If $\left(\chi_{k \alpha}\right)^{\tilde{2}}$ denotes the $D$-comodule structure induced by $\chi_{k \alpha}$ on $H^{k}(D, \alpha(M))$, Theorem 3.7 guarantees that $\left(\chi_{k \alpha}\right) \tilde{)}(t)=t \otimes 1_{D}$, for $t \in H^{k}(D, \alpha(M))$. If $\tilde{\chi}_{k}$ denotes the $C$-structure defined by $\chi_{k}$ on $H^{k}(D, M)$, it is clear from our definitions that $\left(\tilde{\chi}_{k}\right)_{\alpha}=$ $\left(\chi_{k \alpha}\right) \tilde{)}$. The above considerations amount to the fact that, for every $t \in H^{k}(D, \alpha(M))$, we have $\left(\tilde{\chi}_{k}\right)_{\alpha}(t)=t \otimes 1_{D}$. 
TheOREM 3.8. Let $C$ and $D$ be Hopf Algebras over a field $k$, let $\theta$ be an adjoint $C$-structure on $D$ and let $\alpha$ be a functor compatible with the structure $\theta$. Assume also that the map $U: C \rightarrow D$ defined as above is surjective. Then $\alpha(C)^{D}$ is a subcoalgebra of $C$ and for every $C$-comodule $M$ there is a natural $\alpha(C)^{D}$ comodule structure $\tilde{\chi}_{k}$ on $H^{k}(D, \alpha(M))$ such that

(a) $\tilde{\chi}_{0}$ coincides with $\chi_{M}$.

(b) For every exact sequence of $C$-comodules $0 \rightarrow M \rightarrow N \rightarrow S \rightarrow 0$ all the maps of the sequence

$$
\begin{aligned}
\cdots & \rightarrow H^{i}(D, \alpha(M)) \rightarrow H^{i}(D, \alpha(N)) \rightarrow H^{i}(D, \alpha(S)) \\
& \rightarrow H^{i+1}(D, \alpha(M)) \rightarrow \cdots
\end{aligned}
$$

are $\alpha(C)^{D}$ comodule maps.

Proof. The only assertions requiring a proof (see Theorem 3.6) are

(i) $\alpha(C)^{D}$ is a subcoalgebra of $C$.

(ii) $\tilde{\chi}_{l}\left(H^{i}(D, \alpha(M))\right) \subset H^{i}(D, \alpha(M)) \otimes \alpha(C)^{D}$.

Clearly (i) is a particular case of (ii) with $M=C$ and $i=0$. Now consider $x \in H^{i}(D, \alpha(M))$. By Lemma 3.3(b), we have $\left(\tilde{\chi}_{k} \otimes \mathrm{id}\right)\left(\tilde{\chi}_{k}\right)_{\alpha}=$ $\left(\right.$ id $\left.\otimes \Delta_{\alpha}\right) \tilde{\chi}_{k}$. If $\tilde{\chi}_{k}(x)=\sum x_{i} \otimes c_{i}$, then, since $\left(\tilde{\chi}_{k}\right)_{\alpha}(x)=x \otimes 1$ (see comments after Theorem 3.7), we have $\sum x_{i} \otimes c_{i} \otimes 1=\sum x_{i} \otimes \Delta_{\alpha}\left(c_{i}\right)$. Since we are working over a field, we may conclude that $\tilde{\chi}_{k}(x) \in$ $H^{k}(D, \alpha(M)) \otimes \alpha(C)^{D}$.

LEMMA 3.9. In the situation above, $\left(\alpha(M)^{D}\right)^{\alpha(C)^{D}}=M^{C}$.

Proof. It is clear that $\left(\alpha(M)^{D}\right)^{\alpha(C)^{D}} \subset M^{C}$. Our result will be proved as soon as we have shown that $M^{C} \subset \alpha(M)^{D}$. Using Lemma 3.3, part (b), we deduce that if $m \in M^{C}$, then $m \otimes 1 \otimes 1=\left(\chi_{M} \otimes \mathrm{id}\right) \chi_{M \alpha}(m)$. Applying (id $\otimes \varepsilon \otimes \mathrm{id}$ ) we obtain $m \otimes 1=\chi_{M \alpha}(m)$.

Definition 3.10. Let $C$ be a Hopf Algebra defined over a ring $R$ and assume that the antipode of $C$ is involutory. The map $\theta: C \rightarrow C \otimes C$, $\theta=(\eta \otimes \mu)(\Delta \eta \otimes \mathrm{id}) \Delta$ is called the conjugate comodule structure on $C$.

As $\Delta \eta=s(\eta \otimes \eta) \Delta$ and $\eta^{2}=$ id, we have

$$
\theta=(\mathrm{id} \otimes \mu)(\mathrm{id} \otimes \eta \otimes \mathrm{id})(s \otimes \mathrm{id})(\Delta \otimes \mathrm{id}) \Delta .
$$

Definition 3.11. Let $C$ and $D$ be Hopf Algebras defined over a ring $R$, and assume that the antipode of $C$ is involutory. Let $\pi: C \rightarrow D$ be a surjective bialgebra map. We say that $\pi$ is normal if $\theta(\operatorname{Ker} \pi) \subset(\operatorname{ker} \pi \otimes$ $C$ ) where $\theta$ is the conjugate structure defined above. 
In the situation of Definition 3.11 there is a unique map $\tilde{\theta}$ that makes the diagram that follows commutative.

$$
\begin{array}{lll}
C & \stackrel{\theta}{\rightarrow} & C \otimes C \\
\downarrow \pi & & \downarrow \pi \otimes \mathrm{id} \\
D & \stackrel{\tilde{\theta}}{\rightarrow} & D \otimes C
\end{array}
$$

Lemma 3.12. Let $C, D, \theta, \tilde{\theta}$ and $\pi$ as above. Then $\tilde{\theta}$ is an adjoint $C$-structure on $D$, in the sense of Definition 3.1.

Proof. Using the fact that $\pi$ is a bialgebra map it is easy to reduce that Lemma to the case $C=D, \pi=\mathrm{id}, \theta=\tilde{\boldsymbol{\theta}}$. In that case, we have to prove

(a) $\theta$ is a $C$-comodule map.

(b) $\theta u=u \otimes u$.

(c) $(\Delta \otimes \mathrm{id}) \theta=(\theta \otimes \theta) \Delta$.

(d) $(\varepsilon \otimes \varepsilon) \theta=\varepsilon$.

The verification of (b) and (d) is more or less immediate. As to (a) and (c), we shall verify (a). The verification of (c) is similar but longer. We leave if for the patient reader. We check that $(\mathrm{id} \otimes \Delta) \theta=(\theta \otimes \mathrm{id}) \theta$. Using the definition of $\theta$ and the equalities $\Delta \eta=s(\eta \otimes \eta) \Delta$,

$$
\Delta \mu=(\mu \otimes \mu)(\mathrm{id} \otimes s \otimes \mathrm{id})(\Delta \otimes \Delta),
$$

we have

$(\mathrm{id} \otimes \Delta) \theta=(\mathrm{id} \otimes \Delta)(\mathrm{id} \otimes \mu)(\mathrm{id} \otimes \eta \otimes \mathrm{id})(s \otimes \mathrm{id})(\Delta \otimes \mathrm{id}) \Delta$

$=(\mathrm{id} \otimes \mu \otimes \mu)(\mathrm{id} \otimes \mathrm{id} \otimes s \otimes \mathrm{id})(\mathrm{id} \otimes \Delta \otimes \Delta)$

$(\mathrm{id} \otimes \eta \otimes \mathrm{id})(s \otimes \mathrm{id})(\Delta \otimes \mathrm{id}) \Delta$

$=(\mathrm{id} \otimes \mu \otimes \mu)(\mathrm{id} \otimes \mathrm{id} \otimes s \otimes \mathrm{id})(\mathrm{id} \otimes s \otimes \mathrm{id} \otimes \mathrm{id})$

$(\mathrm{id} \otimes \eta \otimes \eta \otimes \mathrm{id} \otimes \mathrm{id})\left(s_{12,3} \otimes \mathrm{id} \otimes \mathrm{id}\right)(\Delta \otimes \mathrm{id} \otimes \Delta)(\Delta \otimes \mathrm{id}) \Delta$.

Similarly

$(\theta \otimes \mathrm{id}) \theta=(\mathrm{id} \otimes \mu \otimes \mu)(\mathrm{id} \otimes \eta \otimes \mathrm{id} \otimes \mathrm{id} \otimes \mathrm{id})$

$(s \otimes \mathrm{id} \otimes \mathrm{id} \otimes \mathrm{id})(\mathrm{id} \otimes \mathrm{id} \otimes \mathrm{id} \otimes \eta \otimes \mathrm{id})$

$(\Delta \otimes \mathrm{id} \otimes \mathrm{id} \otimes \mathrm{id})(\Delta \otimes \mathrm{id} \otimes \mathrm{id})(s \otimes \mathrm{id})(\Delta \otimes \mathrm{id}) \Delta$. 
A direct computation with $(\Delta \otimes \mathrm{id} \otimes \mathrm{id} \otimes \mathrm{id})(\Delta \otimes \mathrm{id} \otimes \mathrm{id})(s \otimes \mathrm{id})$ gives $(\theta \otimes \mathrm{id}) \theta=(\mathrm{id} \otimes \mu \otimes \mu)(\mathrm{id} \otimes \eta \otimes \mathrm{id} \otimes \mathrm{id} \otimes \mathrm{id})$

$$
\begin{aligned}
& (s \otimes \mathrm{id} \otimes \mathrm{id} \otimes \mathrm{id})(\mathrm{id} \otimes \mathrm{id} \otimes \mathrm{id} \otimes \eta \otimes \mathrm{id}) \\
& \left(s_{1,234} \otimes \mathrm{id}\right)(\mathrm{id} \otimes \Delta \otimes \mathrm{id} \otimes \mathrm{id})(\mathrm{id} \otimes \Delta \otimes \mathrm{id})(\Delta \otimes \mathrm{id}) \Delta .
\end{aligned}
$$

By coassociativity,

$(\Delta \otimes \mathrm{id} \otimes \Delta)(\Delta \otimes \mathrm{id}) \Delta=(\mathrm{id} \otimes \Delta \otimes \mathrm{id} \otimes \mathrm{id})(\mathrm{id} \otimes \Delta \otimes \mathrm{id})(\Delta \otimes \mathrm{id}) \Delta$.

Therefore, in order to prove that $(\theta \otimes \mathrm{id}) \theta=(\mathrm{id} \otimes \Delta) \theta$, all we have to verify is that

$$
\begin{gathered}
(\mathrm{id} \otimes \mu \otimes \mu)(\mathrm{id} \otimes \eta \otimes \mathrm{id} \otimes \mathrm{id} \otimes \mathrm{id})(s \otimes \mathrm{id} \otimes \mathrm{id} \otimes \mathrm{id}) \\
(\mathrm{id} \otimes \mathrm{id} \otimes \mathrm{id} \otimes \eta \otimes \mathrm{id})\left(s_{1,234} \otimes \mathrm{id}\right) \\
=(\mathrm{id} \otimes \mu \otimes \mu)(\mathrm{id} \otimes \mathrm{id} \otimes s \otimes \mathrm{id})(\mathrm{id} \otimes s \otimes \mathrm{id} \otimes \mathrm{id}) \\
(\mathrm{id} \otimes \eta \otimes \eta \otimes \mathrm{id} \otimes \mathrm{id})\left(s_{12,3} \otimes \mathrm{id} \otimes \mathrm{id}\right) .
\end{gathered}
$$

This equality can be verified directly.

Now for arbitrary coalgebras, we can make the following definition.

Definition 3.13. Let $C$ and $D$ be coalgebras defined over $R$, a commutative ring with identity, and assume that $C$ and $D$ are flat as $R$-modules. Let $\pi$ be a surjective coalgebra map from $C$ onto $D$. The map $\pi$ induces a functor $\pi_{*}$ : $\operatorname{eg}(C) \rightarrow \operatorname{eg}(D)$ as follows:

(a) If $\left(M, \chi_{M}\right)$ is an object in $\operatorname{Cor}(C)$,

$$
\pi_{*}\left(M, \chi_{M}\right)=\left(M,(\mathrm{id} \otimes \pi) \chi_{M}\right) .
$$

(b) If $f: M \rightarrow N$ is a $C$-comodule map $\pi_{*}(f)=f$.

There are a few things to verify in the definition above, all of which are immediate.

Lemma 3.14. Let $C, D, \pi, \theta$, and $\tilde{\theta}$ be as in Lemma 3.12. Then, the functor $\pi_{*}$ is compatible with the adjoint structure $\tilde{\theta}$.

Proof. Referring to definition 3.2, the only condition not immediately clear is condition (e). Again an easy argument reduces our verification to the case $C=D, \pi=$ id, $\theta=\tilde{\theta}$, in which case condition (e) of Definition 3.2 reads: $\left(\chi_{M} \otimes \theta\right) \chi_{M}=\left(\chi_{M} \otimes\right.$ id $) \chi_{M}$. Actually, we have

$$
\begin{aligned}
\left(\chi_{M}\right. & \otimes \theta) \chi_{M} \\
& =(\mathrm{id} \otimes \mathrm{id} \otimes \mu)(\mathrm{id} \otimes s \otimes \mathrm{id})(\mathrm{id} \otimes \mathrm{id} \otimes \theta)\left(\chi_{M} \otimes \mathrm{id}\right) \chi_{M} \\
& =(\mathrm{id} \otimes \mathrm{id} \otimes \mu)(\mathrm{id} \otimes s \otimes \mathrm{id})(\mathrm{id} \otimes \mathrm{id} \otimes \theta)(\mathrm{id} \otimes \Delta) \chi_{M} .
\end{aligned}
$$


As $\left(\chi_{M} \otimes \mathrm{id}\right) \chi_{M}=(\mathrm{id} \otimes \Delta) \chi_{M}$, it is enough to prove that

$$
(\mathrm{id} \otimes \mu)(s \otimes \mathrm{id})(\mathrm{id} \otimes \theta) \Delta=\Delta .
$$

If we write down the definition of $\theta$ in the formula above we obtain

$$
\begin{aligned}
& (\mathrm{id} \otimes \mu)(s \otimes \mathrm{id})(\mathrm{id} \otimes \theta) \Delta \\
& \quad=(\mathrm{id} \otimes \mu)(s \otimes \mathrm{id})(\mathrm{id} \otimes \mathrm{id} \otimes \mu)(\mathrm{id} \otimes s \otimes \mathrm{id})(\mathrm{id} \otimes \eta \otimes \mathrm{id} \otimes \mathrm{id}) \\
& \quad(\mathrm{id} \otimes \Delta \otimes \mathrm{id})(\mathrm{id} \otimes \Delta) \Delta \\
& =(\mathrm{id} \otimes \mu)(s \otimes \mathrm{id})(\mu(\mathrm{id} \otimes \eta) \Delta \otimes \mathrm{id} \otimes \mathrm{id})(\mathrm{id} \otimes \Delta) \Delta .
\end{aligned}
$$

As

$\mu(\mathrm{id} \otimes \eta) \Delta=u \varepsilon \quad$ and $\quad(\mathrm{id} \otimes \mu)(s \otimes \mathrm{id})(u \varepsilon \otimes \mathrm{id} \otimes \mathrm{id})(\mathrm{id} \otimes \Delta)=\mathrm{id}$, we deduce our result.

In the case of Lemma $3.14, \Delta_{\pi_{*}}=(\mathrm{id} \otimes \pi) \Delta$. Then the map $U$ considered in Theorem 3.8 coincides with $\pi$ and consequently is surjective. Therefore, Theorem 3.8 yields the following result:

THeOREM 3.15. Let $C$ and $D$ be Hopf Algebras over a field, that have involutory antipodes, and let $\pi$ be a surjective normal bialgebra map from $C$ to $D$. Then, given any $C$-comodule $M$ there is a natural $\pi_{*}(C)^{D}$ comodule structure $\tilde{\chi}_{k}$ on $H^{k}\left(D, \pi_{*}(M)\right)$ such that:

(a) $\tilde{\chi}_{0}$ coincides with $\chi_{M}$.

(b) If $0 \rightarrow M \rightarrow N \rightarrow S \rightarrow 0$ is an exact sequence of $C$-comodules, the maps in the sequence

$$
\begin{aligned}
\cdots & \rightarrow H^{k}\left(D, \pi_{*}(M)\right) \rightarrow H^{k}\left(D, \pi_{*}(N)\right) \rightarrow H^{k}\left(D, \pi_{*}(S)\right) \\
& \rightarrow H^{k+1}\left(D, \pi_{*}(M)\right) \rightarrow \cdots
\end{aligned}
$$

are $\pi_{*}(C)^{D}$ comodule maps.

4. Induced comodules and injective comodules. In this section we shall study cetain injective comodules in order to be able to prove that the spectral sequences of $\S 5$ converge. We shall generalize results on injective comodules from $[4,5][1]$ and $[3]$.

LeMma 4.1. Let $C$ be a Hopf Algebra over a field, let $M$ be an arbitrary $C$-comodule and let $V$ be a finite dimensional $C$-comodule. If we endow $V^{*}$ (dual space of $V$ ) with its dual C-comodule structure then $\left(M \otimes V^{*}\right)^{C}=$ $\operatorname{Hom}_{C}(V, M)$. 
Proof. Let us choose a basis of $V,\left(v_{i}\right) i=1, \ldots, n$, and let $\left(f_{i}\right)$ be the dual basis of $V^{*}$. If $\chi_{V}\left(v_{j}\right)=\Sigma_{k} v_{k} \otimes c_{j k}$ the dual $C$-comodule structure on $V^{*}$ is given by $\chi_{V^{*}}\left(f_{i}\right)=\Sigma_{k} f_{k} \otimes \eta\left(c_{k i}\right)$. Consider the $k$-linear isomorphism $\Phi: \quad M \otimes V^{*} \rightarrow \operatorname{Hom}_{k}(V, M)$ given by $\Phi\left(\sum_{i} m_{i} \otimes \gamma_{i}\right)(v)=$ $\Sigma_{l} \gamma_{i}(v) m_{i}$. We shall prove that $\Phi\left(\left(M \otimes V^{*}\right)^{C}\right)=\operatorname{Hom}_{C}(V, M)$. The condition for an element $\Sigma_{i} m_{i} \otimes f_{i}$ to be in $\left(M \otimes V^{*}\right)^{C}$ can be expressed as follows, where $\chi_{M}\left(m_{i}\right)=\Sigma_{t} m_{t i} \otimes c_{t i}^{\prime}$.

$$
\sum_{i} m_{t} \otimes f_{i} \otimes 1_{C}=\sum_{i k t} m_{t i} \otimes f_{k} \otimes c_{t i}^{\prime} \eta\left(c_{k i}\right) .
$$

Thus, $\sum m_{i} \otimes f_{i} \in\left(M \otimes V^{*}\right)^{C}$ if and only if the following condition is satisfied:

$$
m_{k} \otimes 1_{C}=\sum_{i t} m_{t i} \otimes c_{t i}^{\prime} \eta\left(c_{k i}\right) \text { for } k=1, \ldots, n .
$$

The condition $\Phi\left(\sum m_{i} \otimes f_{i}\right) \in \operatorname{Hom}_{C}(V, M)$ means that the maps $\chi_{M}\left(\Phi\left(\sum m_{i} \otimes f_{i}\right)\right)$ and $\left(\Phi\left(\sum m_{i} \otimes f_{i}\right) \otimes \mathrm{id}\right) \chi_{V}$ from $V$ to $M \otimes C$ coincide. This happens if and only if they coincide at $v_{j}$ for $j=1, \ldots, n$. As $\Phi\left(\sum m_{i} \otimes f_{i}\right)\left(v_{j}\right)=m_{j}$, the maps coincide if and only if $\chi_{M}\left(m_{j}\right)=$ $\Sigma_{k} m_{k} \otimes c_{j k}$. Thus $\Phi\left(\sum m_{i} \otimes f_{i}\right) \in \operatorname{Hom}_{C}(V, M)$ if and only if the following condition is satisfied:

$$
\sum_{t} m_{t j} \otimes c_{t_{j}}^{\prime}=\sum_{k} m_{k} \otimes c_{j k}
$$

As $c_{J k}$ are the matrix coefficients of a comodule structure, they satisfy

$$
\Delta\left(c_{j t}\right)=\sum_{k t} c_{k t} \otimes c_{j k} \quad \text { and } \quad \varepsilon\left(c_{j k}\right)=\delta_{j k} .
$$

Hence,

$$
\sum_{k} c_{k t} \eta\left(c_{j k}\right)=\mu(\mathrm{id} \otimes \eta) \Delta\left(c_{j t}\right)=u\left(\varepsilon\left(c_{j t}\right)\right)=\delta_{j t}
$$

and

$$
\sum_{k} \eta\left(c_{k t}\right) c_{j k}=\delta_{j t}
$$

If we tensor $(* *)$ with $c_{r j}$, apply id $\otimes$ id $\otimes \eta$ and multiply, we obtain

$$
\begin{aligned}
\sum_{j t} m_{j t} \otimes c_{t j}^{\prime} \eta\left(c_{r j}\right) & =\sum_{k j} m_{i} \otimes c_{j k} \eta\left(c_{r j}\right) \\
& =\sum_{k} m_{k} \otimes \sum_{j} c_{j k} \eta\left(c_{r j}\right) \\
& =\sum_{k} m_{k} \otimes \delta_{r k}=m_{r} \otimes 1_{C} .
\end{aligned}
$$


This is exactly condition $(*)$. In a similar way we can go from $(*)$ to $(* *)$.

THeOREM 4.2. Let $C$ be a Hopf Algebra over a field with involutory antipode. Let $M$ be an arbitrary $C$-comodule, and let $F_{M}$ denote the functor from $\operatorname{CN}(C)$ to $\Re(k)$ given by $F_{M}(V)=(M \otimes V)^{C}$. Then $M$ is injective as a $C$-comodule if and only if the functor $F_{M}$ is exact. (Note that the functor $F_{M}$ is always left exact.)

Proof. It is easy to see (using a Zorn's Lemma type of argument) that $M$ is injective as a $C$-comodule if and only if for every pair $\left(V_{1}, V_{2}\right)$ of finite dimensional $C$-comodules, for every injective $C$-comodule map $\alpha: V_{1} \rightarrow V_{2}$ and for every $C$-comodule map $f: V_{1} \rightarrow M$, there exists a $C$-comodule map $\tilde{f}: V_{2} \rightarrow M$ such that $\tilde{f} \alpha=f$.

In a similar fashion we can reduce the proof that $F_{M}$ is right exact to the finite dimensional case. Denote $\mathcal{C} \Re_{f}(M)$ the category of finite dimensional $C$-comodules, and let us call $i$ the functor from $\operatorname{Cg} \pi_{f}(C)$ to itself that sends $V$ to $V^{*}$. We have proved that, on $e \pi_{f}(C), F_{M} \dot{i}$ is naturally equivalent to $\operatorname{Hom}_{C}(-, M)$. The $C$-comodule $M$ is injective if and only if the functor $\operatorname{Hom}_{C}(-, M)$ from $\operatorname{CgR}_{f}(C)$ to $\Re(k)$ is left exact. Thus, it is clear that, on $\operatorname{Cg}_{f}(C), F_{M}$ right exact implies that $\operatorname{Hom}_{C}(-, M)$ is left exact. Conversely, as $\eta^{2}=\mathrm{id}, F_{m} \mathrm{i} i$ is naturally equivalent to $F_{M}$ on $\operatorname{e} \pi_{f}(C)$. Consequently, on $\operatorname{C} \pi_{f}(C), F_{M}$ is equivalent to $\operatorname{Hom}_{C}(-, M)$ i. Thus our conclusion follows.

COROLlary 4.3. In the situation of Theorem 4.2, $M$ is an injective $C$-comodule if and only if $H^{i}(C, M \otimes V)=0$ for every $C$-comodule $V$.

Now consider the following situation. Let $C$ and $D$ be coalgebras over a field $k$, and let $\pi: C \rightarrow D$ be a surjective coalgebra map. In $\S 3$, we constructed the functor $\pi_{*}$ : C $\Re(C) \rightarrow \operatorname{CoR}(D)$. We shall see that $\pi_{*}$ has a right adjoint $\pi^{*}$ that is closely related to the functor $F_{M}$ constructed above, for $M=\pi_{*}(C)$.

Let $V$ be a $D$-comodule with structure $\chi_{V}$. We define a map $\Phi_{V}: V \otimes C \rightarrow V \otimes D \otimes C$ by $\Phi_{V}=\left(\chi_{V} \otimes \mathrm{id}_{C}\right)-\mathrm{id}_{V} \otimes(\pi \otimes \mathrm{id}) \Delta_{C}$. It is immediate that, with respect to the $C$-comodule structure id $\otimes \Delta$ on $V \otimes C$, the kernel of $\Phi_{V}$ is a subcomodule. Using the notations of [8], $\operatorname{Ker} \Phi_{V}$ is the cotensor product of the right $D$-comodule $V$ with structure $\chi_{V}$ and the left $D$-comodule $C$ with structure $(\pi \otimes \mathrm{id}) \Delta_{C}$. Another interpretation of the $C$-comodule $\operatorname{Ker} \Phi_{V}$ with structure id $\otimes \Delta$ is the following. The coalgebra structure of $C$ induces an algebra structure on $C^{*}$ (dual 
space of $C$ ). If $V$ is a $C$-comodule, we can associate to it a $C^{*}$-module in the usual fashion. (The base space is the same $V$ and if $v \in V$ and $f \in C^{*}$, $f \cdot v=\Sigma_{i} f\left(c_{l}\right) v_{i}$, where $\chi_{V}(v)=\Sigma c_{l} \otimes v_{l}$.) If $\pi: C \rightarrow D$ is a surjective coalgebra map, it induces an injective algebra map from $D^{*}$ into $C^{*}$. In that case if $V$ is a $D$-comodule, $\operatorname{Ker} \Phi_{V}$ is a $C$-comodule that, with the associated $C^{*}$-module structure, is isomorphic with $\operatorname{Hom}_{D^{*}}\left(C^{*}, V\right)$. Here, we are considering $V$ as a $D^{*}$-module as above and $C^{*}$ as a $D^{*}$-module via the injection from $D^{*}$ into $C^{*}$, and $\operatorname{Hom}_{D^{*}}\left(C^{*}, V\right)$ is endowed with a $C^{*}$-module structure in the usual fashion.

If $C=P(G)$ and $D=P(K)$, with $K$ a closed subgroup of the affine algebraic group $G$, and $\pi$ is the restriction map, the space $\operatorname{Ker} \Phi_{V}$ with the structure (id $\otimes \Delta$ ) is the induced representation functor studied in [1].

It is easy to see that if $f: V \rightarrow W$ is a morphism of $D$-comodules, then $(f \otimes \mathrm{id})\left(\operatorname{Ker} \Phi_{V}\right) \subset \operatorname{Ker} \Phi_{W}$.

DeFinition 4.4. In the situation above, we define the functor $\pi^{*}$ from Cor $(D)$ to e R $(C)$ as follows. If $V$ is a $D$-comodule then $\pi^{*}(V)=$ $\operatorname{Ker} \Phi_{V}$, endowed with the structure $\mathrm{id}_{V} \otimes \Delta$; if $f$ is a $D$-comodule map from $V$ to $W$ then $\pi^{*}(f)$ is the restriction of $f \otimes$ id to $\pi^{*}(V)$.

LeMma 4.5. In the situation above, $\pi^{*}(D)$ is isomorphic with $C$, as a C-comodule.

Proof. Consider the map $\gamma: C \rightarrow D \otimes C$, where $\gamma=(\pi \otimes$ id $) \Delta$. We have

$$
\begin{aligned}
\Phi_{D} \gamma & =((\Delta \otimes \mathrm{id})-\mathrm{id} \otimes(\pi \otimes \mathrm{id}) \Delta)(\pi \otimes \mathrm{id}) \Delta \\
& =(\Delta \pi \otimes \mathrm{id}) \Delta-(\pi \otimes \pi \otimes \mathrm{id})(\mathrm{id} \otimes \Delta) \Delta \\
& =(\Delta \pi \otimes \mathrm{id}) \Delta-((\pi \otimes \pi) \Delta \otimes \mathrm{id}) \Delta=0 .
\end{aligned}
$$

Thus, $\gamma(C) \subset \pi^{*}(D)$. Consider now $\delta: D \otimes C \rightarrow C$, where $\delta(d \otimes c)=$ $\varepsilon(d) c$. The restriction of $\delta$ to $\pi^{*}(D)$ is the inverse of $\gamma$, because if $x \in C$ and $\Delta(x)=\sum x_{l} \otimes x_{l}^{\prime}$ then $(\delta \gamma)(x)=\sum_{l} \varepsilon\left(\pi\left(x_{l}\right)\right) x_{l}^{\prime}=\sum \varepsilon\left(x_{i}\right) x_{l}^{\prime}=x$. Conversely, if $\sum d_{i} \otimes c_{l} \in \pi^{*}(D)$, then

$$
\sum \Delta\left(d_{l}\right) \otimes c_{i}=\sum d_{i} \otimes(\pi \otimes \mathrm{id}) \Delta\left(c_{i}\right) .
$$

Applying $\varepsilon \otimes \mathrm{id} \otimes \mathrm{id}$ we get

$$
\sum d_{l} \otimes c_{l}=\sum \varepsilon\left(d_{l}\right)(\pi \otimes \mathrm{id}) \Delta\left(c_{l}\right)=(\gamma \delta)\left(\sum d_{l} \otimes c_{l}\right) .
$$


Finally, $\gamma$ is a $C$-map because

$$
\begin{aligned}
(\mathrm{id} \otimes \Delta) \gamma & =(\mathrm{id} \otimes \Delta)(\pi \otimes \mathrm{id}) \Delta=(\pi \otimes \mathrm{id} \otimes \mathrm{id})(\mathrm{id} \otimes \Delta) \Delta \\
& =(\pi \otimes \mathrm{id} \otimes \mathrm{id})(\Delta \otimes \mathrm{id}) \Delta=(\gamma \otimes \mathrm{id}) \Delta .
\end{aligned}
$$

If $V$ is a $D$-comodule, we consider the map $E_{V}: \pi^{*}(V) \rightarrow V$ given by $E_{V}\left(\sum v_{i} \otimes c_{l}\right)=\sum v_{i} \varepsilon\left(c_{l}\right)$.

LEMMA 4.6. (a) If $V$ is a D-comodule then $E_{V}$ is a morphism of $D$-comodules from $\pi_{*}\left(\pi^{*}(V)\right)$ to $V$.

(b) If $W$ is a C-comodule, $V$ a $D$-comodule and $f: \pi_{*}(W) \rightarrow V$ a $D$-comodule map, there is one and only one morphism of $C$-comodules $\tilde{f}: W \rightarrow \pi^{*}(V)$ such that $E_{V} \pi_{*}(\tilde{f})=f$.

Proof. (a) If $\sum v_{i} \otimes c_{i}$ verifies $\sum \chi_{V}\left(v_{i}\right) \otimes c_{i}=\sum v_{i} \otimes(\pi \otimes \mathrm{id}) \Delta\left(c_{l}\right)$ when we apply id $\otimes$ id $\otimes \varepsilon$, we have

$$
\sum \varepsilon\left(c_{i}\right) \chi_{V}\left(v_{l}\right)=\sum v_{i} \otimes \pi\left(c_{i}\right) .
$$

Now, if $\Delta\left(c_{l}\right)=\sum c_{\imath k} \otimes c_{l k}^{\prime}$, we have

$$
\begin{aligned}
\left(E_{V}\right. & \otimes \mathrm{id})(\mathrm{id} \otimes \mathrm{id} \otimes \pi)(\mathrm{id} \otimes \Delta)\left(\sum_{l} v_{l} \otimes c_{i}\right) \\
& =\sum_{i k}\left(E_{V} \otimes \mathrm{id}\right)\left(v_{i} \otimes c_{l k} \otimes \pi\left(c_{l k}^{\prime}\right)\right) \\
& =\sum_{i k} \varepsilon\left(c_{i k}\right) v_{i} \otimes \pi\left(c_{i k}^{\prime}\right)=\sum_{i} v_{l} \otimes \sum_{k} \varepsilon\left(c_{i k}\right) \pi\left(c_{i k}^{\prime}\right) \\
& =\sum_{i} v_{i} \otimes \pi\left(c_{i}\right) .
\end{aligned}
$$

Using equality $(*)$ we deduce that

$$
\begin{aligned}
\left(E_{V}\right. & \otimes \mathrm{id})(\mathrm{id} \otimes \mathrm{id} \otimes \pi)(\mathrm{id} \otimes \Delta)\left(\sum v_{i} \otimes c_{l}\right) \\
& =\sum \varepsilon\left(c_{i}\right) \chi_{V}\left(v_{l}\right)=\left(\chi_{V} E_{V}\right) \sum\left(v_{i} \otimes c_{l}\right) .
\end{aligned}
$$

(b) Consider $\tilde{f}: W \rightarrow V \otimes C$ defined by $\tilde{f}=(f \otimes \mathrm{id}) \chi_{W}$. We have

$$
\begin{aligned}
& \Phi_{V}(f \otimes \mathrm{id}) \chi_{W} \\
& \quad=\left(\chi_{V} f \otimes \mathrm{id}\right) \chi_{W}-(f \otimes \mathrm{id} \otimes \mathrm{id})(\mathrm{id} \otimes \pi \otimes \mathrm{id})(\mathrm{id} \otimes \Delta) \chi_{W} .
\end{aligned}
$$

As $f$ is a morphism of $D$-comodules, we have $\chi_{V} f=(f \otimes \mathrm{id})(\mathrm{id} \otimes \pi) \chi_{W}$. Hence, $\Phi_{V}(f \otimes$ id $) \chi_{W}=0$ and $\tilde{f}(W) \subset \pi^{*}(V)$.

It is almost immediate that $E_{V}(f \otimes \mathrm{id}) \chi_{W}=f$. The verification that $\tilde{f}$ is a $C$-comodule map goes along similar lines as the proof of Lemma 4.5. 
It only remains to be proven that if $h: W \rightarrow \pi^{*}(V)$ is a $C$-comodule map such that $E_{V} \pi_{*}(h)=0$, then $h=0$. We have $(h \otimes$ id $) \chi_{W}=($ id $\otimes \Delta) h$. Applying (id $\otimes \varepsilon \otimes \mathrm{id})$, we obtain

$$
((\mathrm{id} \otimes \varepsilon) h \otimes \mathrm{id}) \chi_{W}=(\mathrm{id} \otimes(\varepsilon \otimes \mathrm{id}) \Delta) h .
$$

If $h(x)=\sum v_{i} \otimes c_{i}$ we deduce from the last equality that

$$
((\mathrm{id} \otimes \varepsilon) h \otimes \mathrm{id}) \chi_{W}(x)=\sum v_{i} \otimes 1 \otimes c_{i} .
$$

As $E_{V} \pi_{*} h=0$ we deduce that the left hand side is zero when we identify $V \otimes R$ with $V$ and (id $\otimes \varepsilon$ ) $h$ with $E_{V} \pi_{*}(h)$. In these terms, the right hand side becomes $\sum v_{i} \otimes c_{i}$. Thus we have $h(x)=0$.

\section{COROLlaRY 4.7. $\pi^{*}$ is a right adjoint of $\pi_{*}$.}

In the situation of Definition 4.4, we can consider the functor $F_{\pi_{*}}(C)$ from $\operatorname{CN}(D)$ to $\Re(k)$. Now, $F_{\pi_{*}(C)}(V)=\left(\pi_{*}(C) \otimes V\right)^{D}$, and $\pi_{*}(C) \otimes V$ has a natural right $C$-comodule structure given by

$$
\Delta \otimes \mathrm{id}_{V}: \pi_{*}(C) \otimes V \rightarrow C \otimes \pi_{*}(C) \otimes V .
$$

We shall verify that, when $\pi_{*}(C) \otimes V$ is endowed with this right $C$-comodule structure, then $\left(\pi_{*}(C) \otimes V\right)^{D}$ is a right $C$-subcomodule of $\pi_{*}(C) \otimes$ $V$. Consider $(\Delta \otimes \mathrm{id} \otimes \mathrm{id})\left((\mathrm{id} \otimes \pi) \Delta \otimes \chi_{V}\right)$. By direct computation one finds that

$$
\begin{aligned}
& (\Delta \otimes \mathrm{id} \otimes \mathrm{id})\left((\mathrm{id} \otimes \pi) \Delta \otimes \chi_{V}\right) \\
& \quad=\left(\mathrm{id} \otimes(\mathrm{id} \otimes \pi) \Delta \otimes \chi_{V}\right)(\Delta \otimes \mathrm{id}) .
\end{aligned}
$$

It is clear then that if

$$
\left((\mathrm{id} \otimes \pi) \Delta \otimes \chi_{V}\right)(x)=x \otimes 1,
$$

then

$$
(\Delta \otimes \mathrm{id})(x) \otimes 1=\left(\mathrm{id} \otimes(\mathrm{id} \otimes \pi) \Delta \otimes \chi_{V}\right)(\Delta \otimes \mathrm{id})(x) .
$$

Consequently, $\left(\pi_{*}(C) \otimes V\right)^{D}$ has a natural structure of right $C$-comodule.

Now assume that $C$ is a Hopf Algebra. We know that, using the antipode of $C$, we can transform any right $C$-comodule structure $\chi_{M}$ on $M$ into a left $C$-comodule structure $\tilde{\chi}_{M}: M \rightarrow M \otimes C$, where

$$
\tilde{\chi}_{M}=(\mathrm{id} \otimes \eta) s \chi_{M} \text {. }
$$

In that fashion, when $C$ is a Hopf Algebra, $\left(\pi_{*}(C) \otimes V\right)^{D}$ has a natural left $C$-comodule structure given by (id $\otimes \mathrm{id} \otimes \eta) s_{1,23}\left(\Delta \otimes \mathrm{id}_{V}\right)$. 
Lemma 4.8. Let $C$ and $D$ be Hopf Algebras over a field, each having involutory antipode, and let $\pi: C \rightarrow D$ be a surjective bialgebra map. Then the functors $\pi^{*}$ : $\operatorname{CgR}(D) \rightarrow \operatorname{CgR}(C)$ and $F_{\pi_{*}(C)}$ : $\operatorname{CN}(D) \rightarrow \operatorname{Cg}(C)$ are naturally equivalent.

Proof. Given an arbitrary $D$-comodule $M$, consider the map $($ id $\otimes \eta) s: C \otimes M \rightarrow M \otimes C$. Clearly (id $\otimes \eta) s$ is bijective. We want to verify:

(a) $($ id $\otimes \eta) s\left(\left(\pi_{*}(C) \otimes M\right)^{D}\right)=\operatorname{Ker} \Phi_{M}=\pi^{*}(M)$.

(b) $($ id $\otimes \eta) s$ is a $C$-comodule map when we endow $\left(\pi_{*}(C) \otimes M\right)^{D}$ with the structure (id $\otimes \mathrm{id} \otimes \eta) s_{1,23}\left(\Delta \otimes \mathrm{id}_{\mathrm{M}}\right)$ and $\pi^{*}(M)$ with the structure $\mathrm{id}_{M} \otimes \Delta$. In order to verify (a), let $x=\sum c_{i} \otimes m_{i} \in\left(\pi_{*}(C) \otimes M\right)^{D}$, write $\chi\left(m_{l}\right)=\Sigma_{j} m_{i j} \otimes d_{i j}$ and $\Delta\left(c_{i}\right)=\Sigma_{k} c_{i k} \otimes c_{i k}^{\prime}$. Then we have $x \in\left(\pi_{*}(C) \otimes M\right)^{D}$ if and only if the following condition is satisfied:

$$
\sum_{i} c_{i} \otimes m_{i} \otimes 1=\sum_{i j k} c_{i k} \otimes m_{i j} \otimes \pi\left(c_{i k}^{\prime}\right) d_{i j}
$$

Analogously, we have (id $\otimes \eta)(x) \in \pi^{*}(M)$ if and only if either one of the following two mutually equivalent equalities hold:

$$
\left\{\begin{array}{l}
\sum_{i} \chi m_{i} \otimes \eta c_{i}=\sum_{i k} \chi_{i k} m_{i} \otimes \pi \eta c_{i k}^{\prime} \otimes \eta c_{i k}, \\
\sum_{i} \chi m_{i} \otimes c_{\imath}=\sum_{\imath k} m_{l} \otimes \pi \eta c_{i k}^{\prime} \otimes c_{\imath k} .
\end{array}\right.
$$

From $(* *)$, applying id $\otimes$ id $\otimes s($ id $\otimes \pi) \Delta$, we deduce

$$
\begin{aligned}
\sum_{i j k} m_{l j} \otimes d_{i j} \otimes \pi\left(c_{i k}^{\prime}\right) \otimes c_{i k} \\
=\sum_{i k} m_{i} \otimes \pi \eta c_{i k}^{\prime} \otimes s(\mathrm{id} \otimes \pi) \Delta c_{i k}
\end{aligned}
$$

Changing the order and multiplying on the left hand side, we deduce that

$$
\begin{aligned}
\sum_{i j k} m_{l j} & \otimes \pi\left(c_{i k}^{\prime}\right) d_{l j} \otimes c_{l k} \\
& =\sum_{i} m_{i} \otimes(\mu s \otimes \mathrm{id})(\pi \eta \otimes s(\mathrm{id} \otimes \pi) \Delta) s \Delta\left(c_{\imath}\right) .
\end{aligned}
$$


By direct computation with the switching operators we get

$$
\begin{aligned}
\sum_{i j k} m_{i j} \otimes \pi\left(c_{i k}^{\prime}\right) d_{i j} \otimes c_{i k} \\
=\sum_{i} m_{i} \otimes s(\mathrm{id} \otimes \mu)((\mathrm{id} \otimes \pi) \Delta \otimes \pi \eta) \Delta\left(c_{i}\right) \\
=\sum_{i} m_{i} \otimes s(\mathrm{id} \otimes \mu)(\mathrm{id} \otimes \pi \otimes \pi) \\
\quad(\mathrm{id} \otimes \mathrm{id} \otimes \eta)(\mathrm{id} \otimes \Delta) \Delta\left(c_{i}\right) \\
=\sum_{i} m_{i} \otimes s(\mathrm{id} \otimes \pi)(\mathrm{id} \otimes \mu(\mathrm{id} \otimes \eta) \Delta) \Delta\left(c_{i}\right) \\
=\sum_{i} m_{i} \otimes s(\mathrm{id} \otimes \pi)\left(c_{\imath} \otimes 1\right) \\
=\sum_{i} m_{i} \otimes 1 \otimes c_{i} .
\end{aligned}
$$

This is exactly the equality $(*)$. In a similar way we can go from $(*)$ to $(* *)$. Establishing (b) amounts to verifying that

$(\mathrm{id} \otimes \Delta)(\mathrm{id} \otimes \eta) s=(\mathrm{id} \otimes \eta \otimes \mathrm{id})(s \otimes \mathrm{id})(\mathrm{id} \otimes \mathrm{id} \otimes \eta) s_{1,23}(\Delta \otimes \mathrm{id})$. Now

$$
\begin{aligned}
(\mathrm{id} & \otimes \\
\quad & \eta(\mathrm{id} \otimes \eta \otimes \mathrm{id})(s \otimes \mathrm{id})(\mathrm{id} \otimes \mathrm{id} \otimes \eta)(\mathrm{id} \otimes \mathrm{id} \otimes \eta)(s \otimes \mathrm{id}) s_{1,23}(\Delta \otimes \mathrm{id}) \\
& =(\mathrm{id} \otimes \eta \otimes \eta)(\mathrm{id} \otimes s)(\mathrm{id} \otimes \Delta) s .
\end{aligned}
$$

The last equality follows from an elementary manipulation with switching maps

$$
\begin{gathered}
(\mathrm{id} \otimes s)(\mathrm{id} \otimes \Delta) s(x \otimes y)=(\mathrm{id} \otimes s)(\mathrm{id} \otimes \Delta)(y \otimes x) \\
=\sum_{i}(\mathrm{id} \otimes s)\left(y \otimes x_{i} \otimes x_{i}^{\prime}\right)=\sum_{i} y \otimes x_{i}^{\prime} \otimes x_{i}
\end{gathered}
$$

and

$$
\begin{gathered}
(s \otimes \mathrm{id}) s_{1,23}(\Delta \otimes \mathrm{id})(x \otimes y)=\sum_{i}(s \otimes \mathrm{id}) s_{1,23}\left(x_{\imath} \otimes x_{i}^{\prime} \otimes y\right) \\
=\sum_{i}(s \otimes \mathrm{id})\left(x_{\imath}^{\prime} \otimes y \otimes x_{i}\right)=\sum_{i} y \otimes x_{i}^{\prime} \otimes x_{i} .
\end{gathered}
$$

Thus,

$(\mathrm{id} \otimes \eta \otimes \mathrm{id})(s \otimes \mathrm{id})(\mathrm{id} \otimes \mathrm{id} \otimes \eta) s_{1,23}(\Delta \otimes \mathrm{id})$

$$
=(\mathrm{id} \otimes \eta \otimes \eta)(\mathrm{id} \otimes s)(\mathrm{id} \otimes \Delta) s=(\mathrm{id} \otimes \Delta \eta) s .
$$


Corollary 4.9. In the situation above, $\pi_{*}(C)$ is injective in Cor $(D)$ if and only if $\pi^{*}$ is an exact functor or, equivalently, if and only if $H^{1}\left(D, \pi_{*}(C) \otimes V\right)=0$ for every $D$-comodule $V$.

We apply this criterion of injectivity in order to prove that, if the map $\pi$ is normal and $C$ and $D$ are commutative, then $\pi_{*}(C)$ is injective. We shall use the following two results from [7].

TheOREM 4.A ([7] page 6). Let $C$ be a commutative Hopf Algebra over a field $k$ and let $X$ be a sub Hopf Algebra of $C$. Then the inclusion $X \hookrightarrow C$ is faithfully flat.

TheORem 4.B ([7] page 17). Let $\pi$ be a surjective normal Hopf Algebra map between a pair of commutative Hopf Algebras $C$ and $D$ defined over a field. Then $\operatorname{Ker} \pi=\left(\operatorname{Ker} \varepsilon \cap \pi_{*}(C)^{D}\right) C$.

Suppose now that $C$ and $D$ are commutative Hopf Algebras over a field $k$ (this implies that the antipode is involutory) and let $\pi$ be a normal surjective Hopf Algebra map between $C$ and $D$. We shall write ${ }^{D} \pi_{*}(C)$ or ${ }^{D} C$ for the set

$$
\{x \in C \mid(\pi \otimes \mathrm{id}) \Delta(x)=1 \otimes x\} .
$$

It is easy to see that $C^{D}={ }^{D} C$ for symmetry reasons. Using the natural structure of $C$ as a ${ }^{D} C$ module, we endow $C \otimes C$ and $D \otimes C$, as well as $V \otimes C$ for any $k$-spaces $V$, with a ${ }^{D} C$-module structure by multiplication in the second tensor factor. It is elementary to verify that, with respect to the structures mentioned above, the map $(\pi \otimes \mathrm{id}) \Delta: C \rightarrow D \otimes C$ is a ${ }^{D} C$-module map. Given the multiplication $\mu: C \otimes C \rightarrow C$, it is clear that if we indicate by $i: C \otimes C \rightarrow C \otimes{ }_{{ }^{D} C} C$ the canonical surjection, there is a map $\tilde{\mu}: C \otimes{ }^{D} C C \rightarrow C$ such that $\tilde{\mu} i=\mu$. We shall denote this new map $\tilde{\mu}$ again by $\mu$ because there will be no danger of confusion. Recall that $\otimes$ always indicates tensor product over $k$. Consider the maps

$$
\Delta_{D} \otimes \mathrm{id}: D \otimes C \rightarrow D \otimes D \otimes C
$$

and

$$
(\pi \otimes \mathrm{id}) \Delta_{C} \otimes_{{ }^{D} C} \mathrm{id}: C \otimes_{{ }^{D} C} C \rightarrow D \otimes C \otimes_{{ }^{D} C} C .
$$


It is easy to prove that these are right $C$-comodule structures. Next, look at the maps

$$
\begin{aligned}
\psi= & (\mathrm{id} \otimes \mu)\left((\pi \otimes \mathrm{id}) \Delta \otimes_{D} \mathrm{id}\right): \\
& C \otimes_{D_{C}} C \stackrel{(\pi \otimes \mathrm{id}) \Delta \otimes D_{C} \mathrm{id}}{\rightarrow} D \otimes C \otimes_{D_{C}} C \stackrel{\mathrm{id} \otimes \mu}{\rightarrow} D \otimes C, \\
\phi_{1}= & i(\mathrm{id} \otimes \mu)(\mathrm{id} \otimes \eta \otimes \mathrm{id})(\Delta \otimes \mathrm{id}): \\
C \otimes & \stackrel{\Delta \otimes \mathrm{id}}{\rightarrow} C \otimes C \otimes C \stackrel{\mathrm{id} \otimes \eta \otimes \mathrm{id}}{\rightarrow} C \otimes C \otimes C \stackrel{\mathrm{id} \otimes \mu}{\rightarrow} C \otimes C \stackrel{i}{\rightarrow} C \otimes_{D^{D} C} C
\end{aligned}
$$

where, as before, $i$ stands for the canonical map $C \otimes C \stackrel{i}{\rightarrow} C \otimes_{D_{C}} C$.

LEMMA 4.10. In the situation above, there is a unique $k$-linear map $\phi_{0}$ that makes the following diagram commutative.

$$
\begin{array}{lll}
C \otimes C & \stackrel{\phi_{1}}{\rightarrow} & C \otimes{ }_{{ } C} C \\
\downarrow \pi \otimes \text { id } & \nearrow \phi_{0} & \\
D \otimes C &
\end{array}
$$

Proof. All we have to verify is that if $t \in \operatorname{Ker} \pi \otimes C$ then $\phi_{1}(t)=0$. Theorem 4.B says that $\operatorname{Ker} \pi=\left(\operatorname{Ker} \varepsilon \cap{ }^{D} C\right) C$. Take an element $\sum x_{k} \otimes y_{k} \in C \otimes C$ such that $x_{k} \in\left(\operatorname{Ker} \varepsilon \cap \cap^{D} C\right)$. We shall verify that $\phi_{1}\left(\sum x_{k} \otimes y_{k}\right)=0$. Let us write $\Delta\left(x_{k}\right)=\Sigma_{i} x_{k l} \otimes x_{k i}^{\prime}$. Then we have $\phi_{1}\left(\sum x_{k} \otimes y_{k}\right)=\Sigma_{k l} x_{k i} \otimes_{D_{C}} \eta\left(x_{k i}^{\prime}\right) y_{k}$. Since $\pi$ is normal $x_{k l} \in{ }^{D} C$ (because $\left.\Delta\left({ }^{D} C\right) \subset{ }^{D} C \otimes{ }^{D} C\right)$. Consequently,

$$
\phi_{1}\left(\sum x_{i} \otimes y_{k}\right)=1 \otimes \sum_{k i} x_{k i} \eta\left(x_{k i}^{\prime}\right) y_{k}=1 \otimes \sum_{k} u \varepsilon\left(x_{k}\right) y_{k}=0 .
$$

THEOREM 4.11. The map $\psi$ is an isomorphism of right D-comodules from $C \otimes{ }^{D} C C$ to $D \otimes C$, whose inverse is the map $\phi_{0}$ defined above.

Proof. First, we show that $\psi$ is a morphism of right $D$-comodules. This amounts to proving that the diagram below is commutative.

$$
\begin{array}{cccc}
C \otimes_{D_{C} C} C \stackrel{(\pi \otimes \mathrm{id}) \Delta \otimes D_{C} \text { id }}{\rightarrow} & D \otimes C \otimes \otimes_{D_{C}} C & \stackrel{\mathrm{id} \otimes \mu}{\rightarrow} & D \otimes C \\
\downarrow(\pi \otimes \mathrm{id}) \Delta \otimes_{D_{C} \text { id }} & & \downarrow \Delta \otimes \mathrm{id}
\end{array}
$$

$D \otimes C \otimes{ }_{D_{C} C} \stackrel{\mathrm{id} \otimes(\pi \otimes \mathrm{id}) \Delta \otimes_{D_{C} \mathrm{id}}}{\rightarrow} D \otimes D \otimes C \otimes \otimes_{{ }^{C} C} C \stackrel{\mathrm{id} \otimes \mathrm{id} \otimes \mu}{\rightarrow} \quad D \otimes D \otimes C$ 
If we add to the above diagram the map

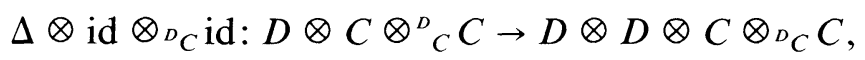

we need only check the commutativity of the two subdiagrams. The resulting square on the left is commutative because $\pi$ is a coalgebra map, and the resulting square on the right is obviously commutative. Next, we show that $\psi$ and $\phi_{0}$ are inverses to each other. Look at

$$
\begin{aligned}
& \psi \phi_{0}(\pi \otimes \mathrm{id})=(\mathrm{id} \times \mu)\left((\pi \otimes \mathrm{id}) \Delta \otimes_{{ }^{D} C} \mathrm{id}\right) \phi_{0}(\pi \otimes \mathrm{id}) \\
& \quad=(\mathrm{id} \otimes \mu)\left((\pi \otimes \mathrm{id}) \Delta \otimes_{{ }^{D} C} \mathrm{id}\right) i(\mathrm{id} \otimes \mu)(\mathrm{id} \otimes \eta \otimes \mathrm{id})(\Delta \otimes \mathrm{id}) .
\end{aligned}
$$

From the very definition of $i$ and $\otimes_{{ }^{D} C}$ we have that

$$
\begin{aligned}
\psi \phi_{0}(\pi \otimes \mathrm{id})= & (\mathrm{id} \otimes \mu)((\pi \otimes \mathrm{id}) \Delta \otimes \mathrm{id})(\mathrm{id} \otimes \mu)(\mathrm{id} \otimes \eta \otimes \mathrm{id})(\Delta \otimes \mathrm{id}) \\
= & (\pi \otimes \mathrm{id})(\mathrm{id} \otimes \mu)(\mathrm{id} \otimes \mu \otimes \mathrm{id})(\mathrm{id} \otimes \mathrm{id} \otimes \eta \otimes \mathrm{id}) \\
& (\mathrm{id} \otimes \Delta \otimes \mathrm{id})(\Delta \otimes \mathrm{id})=\pi \otimes \mathrm{id} .
\end{aligned}
$$

The last equality follows from the fact that $\mu(\mathrm{id} \otimes \eta) \Delta=u \varepsilon$. Since $\pi \otimes \mathrm{id}$ is surjective, we conclude that $\psi \phi_{0}=\mathrm{id}$. Now, we observe, that:

$$
\phi_{0} \psi i=\phi_{0}(\mathrm{id} \otimes \mu)\left((\pi \otimes \mathrm{id}) \Delta \otimes_{D_{C}} \mathrm{id}\right) i=\phi_{0}(\pi \otimes \mathrm{id})(\mathrm{id} \otimes \mu)(\Delta \otimes \mathrm{id}) .
$$

If we replace $\phi_{0}(\pi \otimes \mathrm{id})$ with $\phi_{1}$ and write out $\phi_{1}$ explicitly, we find from an argument similar to one above (relying on the fact that $\eta$ is an antipode) that:

$$
\phi_{0}(\pi \otimes \mathrm{id})(\mathrm{id} \otimes \mu)(\Delta \otimes \mathrm{id})=i .
$$

This shows that $\phi_{0} \psi i=i$. Since the map $i: C \otimes C \rightarrow \otimes{ }^{{ }_{C}} C$ is surjective, we deduce that $\phi_{0} \psi=$ id.

Let $V$ be a $D$-comodule. Then $\pi^{*}(V)$ is a ${ }^{D} C$ submodule of $V \otimes C$ when we endow $V \otimes C$ with the structure of ${ }^{D} C$ module given by multiplication on $C$.

THEOREM 4.12. Let $C$ and $D$ be as before, and let $V$ be a $D$-comodule, then the $k$-linear maps

$$
\text { id } \otimes \mu: V \otimes C \otimes \otimes_{D_{C}} C \rightarrow V \otimes C \text { and } \quad \alpha=\left(\mathrm{id} \otimes \phi_{0}\right)(\chi \otimes \mathrm{id}),
$$

the composite

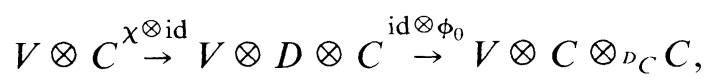


have the following properties:

(a) $\alpha(V \otimes C) \subset \pi^{*}(V) \otimes{ }^{D} C$

(b) $\alpha$ is the inverse of the restriction of id $\otimes \mu$ to $\pi^{*}(V) \otimes_{{ }^{D} C} C$.

Proof. Let us verify first that if $t \in \pi^{*}(V) \otimes_{D_{C}} C$, then

$$
\alpha\left(\operatorname{id}_{V} \otimes \mu\right)(t)=t .
$$

If $t=\sum_{\imath} v_{i} \otimes c_{\imath} \otimes{ }^{D} C \bar{c}_{i}$, then

$$
\sum_{i} \chi_{V}\left(v_{\imath}\right) \otimes c_{i} \otimes{ }^{{ } C} \bar{c}_{\imath}=\sum_{i} v_{i} \otimes(\pi \otimes \mathrm{id}) \Delta\left(c_{i}\right) \otimes{ }^{{ } C} \bar{c}_{i} .
$$

Hence,

$\alpha(\mathrm{id} \otimes \mu)(t)=\left(\mathrm{id} \otimes \phi_{0}\right)(\chi \otimes \mathrm{id})(\mathrm{id} \otimes \mu)(t)$

$$
\begin{aligned}
& =\left(\mathrm{id} \otimes \phi_{0}\right)\left(\sum_{i} \chi\left(v_{i}\right) \otimes c_{i} \bar{c}_{i}\right) \\
& =\left(\mathrm{id} \otimes_{V} \otimes \phi_{0}\right)\left(\sum_{i} v_{i} \otimes(\mathrm{id} \otimes \mu)\right)\left((\pi \otimes \mathrm{id}) \Delta \otimes_{D_{C} C} \mathrm{id}\right)\left(c_{i} \otimes_{D_{C} C} \bar{c}_{i}\right) \\
& =\sum_{i} v_{i} \otimes \phi_{0}(\mathrm{id} \otimes \mu)\left((\pi \otimes \mathrm{id}) \Delta \otimes_{D_{C}} \mathrm{id}\right)\left(c_{i} \otimes_{D_{C}} \bar{c}_{i}\right) \\
& =\sum_{i} v_{i} \otimes \phi_{0} \psi\left(c_{i} \otimes_{D_{C}} \bar{c}_{i}\right)=\sum_{i} v_{\imath} \otimes c_{l} \otimes_{D_{C}} \bar{c}_{i}=t .
\end{aligned}
$$

The equality before the last comes from the fact that $\phi_{0}$ and $\psi$ are mutually inverse maps.

Next, we check that

$$
(\mathrm{id} \otimes \mu) \alpha=\mathrm{id}, \quad(\mathrm{id} \otimes \mu) \alpha=(\mathrm{id} \otimes \mu)\left(\mathrm{id} \otimes \phi_{0}\right)(\chi \otimes \mathrm{id}) .
$$

Now observe that

$$
\begin{aligned}
\mu \phi_{0}(\pi \otimes \mathrm{id}) & =\mu i(\mathrm{id} \otimes \mu)(\mathrm{id} \otimes \eta \otimes \mathrm{id})(\Delta \otimes \mathrm{id}) \\
& =\mu(\mu \otimes \mathrm{id})(\mathrm{id} \otimes \eta \otimes \mathrm{id})(\Delta \otimes \mathrm{id})=\mu(u \varepsilon \otimes \mathrm{id})
\end{aligned}
$$

We have $\mu \phi_{0}(\pi \otimes \mathrm{id})\left(c_{1} \otimes c_{2}\right)=\varepsilon\left(c_{1}\right) c_{2}=j\left(\varepsilon_{D} \otimes \mathrm{id}\right)(\pi \otimes \mathrm{id})\left(c_{1} \otimes c_{2}\right)$, where $j$ stands for the map $j: k \otimes C \rightarrow C$, given by $j(\lambda \otimes c)=\lambda c$. Consequently we have $\mu \phi_{0}=j\left(\varepsilon_{D} \otimes \mathrm{id}\right)$. Hence,

$$
(\mathrm{id} \otimes \mu) \alpha=(\mathrm{id} \otimes j) \quad(\mathrm{id} \otimes \varepsilon \otimes \mathrm{id})(\chi \otimes \mathrm{id})=\mathrm{id}_{\nu \otimes C} .
$$


It remains only to verify (a). We have (recall Definition 4.4)

$$
\begin{aligned}
\left(\phi_{V} \otimes{ }^{D_{C}} \mathrm{id}\right)\left(\mathrm{id} \otimes \phi_{0}\right)(\chi \otimes \mathrm{id}) \\
=\left(\left(\chi \otimes \mathrm{id} \otimes{ }_{D_{C}} \mathrm{id}\right)-\left(\mathrm{id} \otimes(\pi \otimes \mathrm{id}) \Delta \otimes_{{ }^{D} C} \mathrm{id}\right)\right)\left(\mathrm{id} \otimes \phi_{0}\right)(\chi \otimes \mathrm{id}) \\
=\left(\chi \otimes \mathrm{id} \otimes_{D_{C} C} \mathrm{id}\right)\left(\mathrm{id} \otimes \phi_{0}\right)(\chi \otimes \mathrm{id}) \\
\quad-\left(\mathrm{id} \otimes(\pi \otimes \mathrm{id}) \Delta \otimes_{D_{C}} \mathrm{id}\right)\left(\mathrm{id} \otimes \phi_{0}\right)(\chi \otimes \mathrm{id}) .
\end{aligned}
$$

By Theorem $4.11 \phi_{0}$ is a right $D$-comodule map. Therefore,

$$
\left((\pi \otimes \mathrm{id}) \otimes_{{ }^{D} C} \mathrm{id}\right) \phi_{0}=\left(\mathrm{id} \otimes \phi_{0}\right)(\Delta \otimes \mathrm{id})
$$

Hence,

$$
\begin{aligned}
& \left(\phi_{V} \otimes{ }_{D} C \mathrm{id}\right)\left(\mathrm{id} \otimes \phi_{0}\right)(\chi \otimes \mathrm{id}) \\
& =\left(\chi \otimes \mathrm{id} \otimes{ }_{D_{C}} \mathrm{id}\right)\left(\mathrm{id} \otimes \phi_{0}\right)(\chi \otimes \mathrm{id}) \\
& \quad-\left(\mathrm{id} \otimes \mathrm{id} \otimes \phi_{0}\right)(\mathrm{id} \otimes \Delta \otimes \mathrm{id})(\chi \otimes \mathrm{id})=0,
\end{aligned}
$$

because $(\mathrm{id} \otimes \Delta) \chi=(\chi \otimes \mathrm{id}) \chi$.

Thus, $\left(\phi_{V} \otimes_{D_{C}} \mathrm{id}\right) \alpha(V \otimes C)=0$, whence

$$
\alpha(V \otimes C) \subset \operatorname{Ker}\left(\phi_{V} \otimes_{\nu_{C}} \mathrm{id}\right)=\operatorname{Ker}\left(\phi_{V}\right) \otimes_{{ }^{D} C} C,
$$

because $C$ is flat as a ${ }^{D} C$-comodule.

THEOREM 4.13. Let $C$ and $D$ be commutative Hopf Algebras over a field $k$, and let $\pi$ be a surjective normal Hopf Algebra map from $C$ to $D$. Then $\pi_{*}(C)$ is injective as a $D$-comodule.

Proof. By Corollary 4.9, it is enough to prove that if $V_{1}$ and $V_{2}$ are $D$-comodules and $\alpha$ a surjective $D$-comodule map from $V_{1}$ to $V_{2}$, then the map induced by $\alpha$ from $\pi^{*}\left(V_{1}\right)$ to $\pi^{*}\left(V_{2}\right)$ is surjective. As the inclusion ${ }^{D} C \hookrightarrow C$ is faithfully flat (Theorem 4.A), the map $\alpha \otimes$ id is surjective if and only if $\alpha \otimes \mathrm{id} \otimes \otimes_{D_{C}} \otimes \mathrm{id}: \pi^{*}\left(V_{1}\right) \otimes_{{ }^{D} C} C \rightarrow \pi^{*}\left(V_{2}\right) \otimes_{{ }^{D} C} C$, is surjective. The maps $\operatorname{id}_{V_{l}} \otimes \mu: \pi^{*}\left(V_{l}\right) \otimes_{D_{C}} C \rightarrow V_{l} \otimes C$ are isomorphisms and commute with $\alpha$ in the sense that

$$
(\alpha \otimes i d)\left(\mathrm{id}_{V_{1}} \otimes \mu\right)=\left(\mathrm{id}_{V_{2}} \otimes \mu\right)\left(\alpha \otimes \mathrm{id} \otimes_{D_{C}} \mathrm{id}\right) .
$$

Consequently $(\alpha \otimes \mathrm{id})\left(\pi^{*}\left(V_{1}\right)\right)=\pi^{*}\left(V_{2}\right)$ if and only if

$$
\alpha \otimes \mathrm{id}: V_{1} \otimes C \rightarrow V_{2} \otimes C
$$

is surjective. Since $\alpha$ is surjective our conclusion follows. 
Now we have the injectivity results for comodules that we need in order to establish the spectral sequences of $\$ 5$. Before proceeding to do that, we establish one other result that is sometimes useful as a criterion for injectivity, and is a generalization of [5], Proposition 2.2.

Lemma 4.14 is contained in [6], our proof is not different from Sweedler's but is more compatible with our notation. For the next two results our coalgebras need not be flat as $R$-modules.

LEMma 4.14 Let $C$ and $D$ be Hopf Algebras defined over a ring $R$, and let $\pi: C \rightarrow D$ be a bialgebra map. Suppose moreover that there exists a $D$-comodule map $\sigma: D \rightarrow \pi_{*}(C)$. Then the map $Q=\mu(\operatorname{id} \otimes \sigma \pi \eta) \Delta: C \rightarrow C$ sends $C$ into $\pi_{*}(C)^{D}$.

Proof. In convolution notation, we have $Q=\mathrm{id} * \sigma \pi \eta$.

$(\mathrm{id} \otimes \pi) \Delta Q=(\mathrm{id} \otimes \pi) \Delta \mu(\mathrm{id} \otimes \sigma \pi \eta) \Delta$

$$
=(\mu \otimes \mu)(\mathrm{id} \otimes \mathrm{id} \otimes \pi \otimes \pi)(\mathrm{id} \otimes s \otimes \mathrm{id})(\Delta \otimes \Delta)(\mathrm{id} \otimes \sigma \pi \eta) \Delta .
$$

For the last equality we used that $\Delta \mu=(\mu \otimes \mu)($ id $\otimes s \otimes \mathrm{id})(\Delta \otimes \Delta)$ and the fact that $\pi$ is an algebra map.

Thus,

$(\mathrm{id} \otimes \pi) \Delta Q=(\mu \otimes \mu)(\mathrm{id} \otimes s \otimes i d)((\mathrm{id} \otimes \pi) \Delta \otimes(\mathrm{id} \otimes \pi) \Delta \sigma \pi \eta) \Delta$.

As $\sigma$ is a $D$-comodule map, we have

$$
(\mathrm{id} \otimes \pi) \Delta \sigma=(\sigma \otimes \mathrm{id}) \Delta
$$

and

$$
(\mathrm{id} \otimes \pi) \Delta Q=(\mu \otimes \mu)(\mathrm{id} \otimes s \otimes \mathrm{id})((\mathrm{id} \otimes \pi) \Delta \otimes(\sigma \otimes \mathrm{id}) \Delta \pi \eta) \Delta .
$$

Using that $\Delta \pi=(\pi \otimes \pi) \Delta$ and $\Delta \eta=s(\eta \otimes \eta) \Delta$, we get

$$
\begin{aligned}
(\mathrm{id} \otimes \pi) \Delta Q= & (\mu \otimes \mu)(\mathrm{id} \otimes s \otimes \mathrm{id})(\mathrm{id} \otimes \mathrm{id} \otimes s) \\
& (\mathrm{id} \otimes \pi \otimes \pi \otimes \sigma \pi)(\mathrm{id} \otimes \mathrm{id} \otimes \eta \otimes \eta)(\Delta \otimes \Delta) \Delta .
\end{aligned}
$$

Using associativity of $\mu$, coassociativity of $\Delta$ and the fact that $\pi$ is an algebra map, we get

$(\mathrm{id} \otimes \pi) \Delta Q$

$=(\mu \otimes \mathrm{id})(\mathrm{id} \otimes s)(\mathrm{id} \otimes \pi \otimes \sigma \pi)(\mathrm{id} \otimes \mu(\mathrm{id} \otimes \eta) \Delta \otimes \eta)(\mathrm{id} \otimes \Delta) \Delta$

$=(\mu \otimes \mathrm{id})(\mathrm{id} \otimes s)(\mathrm{id} \otimes \pi \otimes \sigma \pi)(\mathrm{id} \otimes u \varepsilon \otimes \eta)(\mathrm{id} \otimes \Delta) \Delta$. 
Now if $\Delta(x)=\sum x_{i} \otimes x_{i}^{\prime}$, we have

$$
\begin{aligned}
(\mathrm{id} \otimes \pi) \Delta Q(x) & =\sum(\mu \otimes \mathrm{id})(\mathrm{id} \otimes s)\left(x_{i} \otimes 1 \otimes \sigma \pi \eta\left(x_{i}^{\prime}\right)\right) \\
& =\sum x_{i} \sigma \pi \eta x_{i}^{\prime} \otimes 1 .
\end{aligned}
$$

But, by the very definition of $Q$, we have $Q x=\sum x_{i} \sigma \pi \eta x_{l}^{\prime}$. Thus, $Q(x) \in \pi_{*}\left(C^{D}\right)$.

THEOREM 4.15. Let $C$ and $D$ be Hopf Algebras over a ring $R$ and let $\pi$ be a bialgebra map from $C$ to $D$. Let $\sigma: D \rightarrow \pi_{*}(C)$ be an algebra map that is at the same time a $D$-comodule homomorphism. Then $\pi_{*}(C)$ is a relatively injective D-comodule.

Proof. Let us consider $C \otimes D$ with structure id $\otimes \Delta$. We shall prove that $\pi_{*}(C)$ is a direct $D$-comodule summand of $C \otimes D$. Consider the maps $\alpha=(\mathrm{id} \otimes \pi) \Delta: C \rightarrow C \otimes D$ and $\beta=\mu(Q \otimes \sigma): C \otimes D \rightarrow C$. We have

$$
\begin{aligned}
\beta \alpha & =\mu(Q \otimes \sigma)(\mathrm{id} \otimes \pi) \Delta=Q * \sigma \pi=(\mathrm{id} * \sigma \pi \eta) * \sigma \pi \\
& =\mathrm{id} *(\sigma \pi \eta * \sigma \pi) .
\end{aligned}
$$

As $\sigma \pi$ is an algebra homomorphism, $\sigma \pi \eta * \sigma \pi=\mu(\sigma \pi \otimes \sigma \pi)(\eta \otimes \mathrm{id}) \Delta=$ $\sigma \pi \mu(\eta \otimes \mathrm{id}) \Delta=\sigma \pi u \varepsilon=u \varepsilon$. Consequently, $\beta \alpha=\mathrm{id} * u \varepsilon=\mathrm{id}$. Now, $\alpha$ is obviously a $D$-map, therefore, the only thing that still requires a proof is that $\beta$ is a $D$-comodule map, i.e. that

$$
(\mathrm{id} \otimes \pi) \Delta \mu(Q \otimes \sigma)=(\mu(Q \otimes \sigma) \otimes \mathrm{id})(\mathrm{id} \otimes \Delta) .
$$

We have,

$$
\begin{aligned}
& (\mathrm{id} \otimes \pi) \Delta \mu(Q \otimes \sigma) \\
& \quad=(\mu \otimes \mu)(\mathrm{id} \otimes s \otimes \mathrm{id})(\mathrm{id} \otimes \pi \otimes \mathrm{id} \otimes \pi)(\Delta \otimes \Delta)(Q \otimes \sigma) \\
& \quad=(\mu \otimes \mu)(\mathrm{id} \otimes s \otimes \mathrm{id})((\mathrm{id} \otimes \pi) \Delta Q \otimes(\mathrm{id} \otimes \pi) \Delta \sigma) .
\end{aligned}
$$

Using that $\sigma$ is a $D$-comodule map, we obtain

$$
\begin{aligned}
& (\mathrm{id} \otimes \pi) \Delta \mu(Q \otimes \sigma) \\
& \quad=(\mu \otimes \mu)(\mathrm{id} \otimes s \otimes \mathrm{id})((\mathrm{id} \otimes \pi) \Delta Q \otimes \sigma \otimes \mathrm{id})(\mathrm{id} \otimes \Delta) .
\end{aligned}
$$

To finish the proof that $\beta$ is a $D$-comodule map, all that needs to be verified is that

$$
(\mu \otimes \mu)(\mathrm{id} \otimes s \otimes \mathrm{id})((\mathrm{id} \otimes \pi) \Delta Q \otimes \sigma \otimes \mathrm{id})=\mu(Q \otimes \sigma) \otimes \mathrm{id} .
$$


We have

$$
\begin{aligned}
& (\mu \otimes \mu)(\mathrm{id} \otimes s \otimes \mathrm{id})((\mathrm{id} \otimes \pi) \Delta Q \otimes \sigma \otimes \mathrm{id})(x \otimes y \otimes z) \\
& \quad=(\mu \otimes \mu)(\mathrm{id} \otimes s \otimes \mathrm{id})(Q(x) \otimes 1 \otimes \sigma y \otimes z) \\
& \quad=Q(x) \sigma y \otimes z .
\end{aligned}
$$

As $(\mu(Q \otimes \sigma) \otimes \mathrm{id})(x \otimes y \otimes z)=Q(x) \sigma(y) \otimes z$, our proof is finished. $\square$

5. Two spectral sequences. First, we establish the validity of the Hochschild-Serre spectral sequence for the cohomology of comodules.

TheOrem 5.1. Let $C$ and $D$ be commutative Hopf Algebras over a field $k$, and let $\pi: C \rightarrow D$ be a surjective normal bialgebra map from $C$ to $D$. For every object $M$ in $\operatorname{Cg}(C)$, there is a third quadrant spectral sequence $\left\{E_{r}^{p, q}\right\}$, natural in $M$, such that

$$
E_{2}^{p, q}=H^{p}\left(\pi_{*}(C)^{D}, H^{q}\left(D, \pi_{*}(M)\right)\right), \quad E_{2}^{p, q} \Rightarrow_{p} H^{p+q}(C, M) .
$$

Here we regard $H^{q}\left(D, \pi_{*}(M)\right)$ as a $\pi_{*}(C)^{D}$-comodule, with the structure established in $\S 3$.

Proof. Consider the functors

$$
F: \operatorname{eg}(C) \rightarrow \operatorname{egn}\left(\pi_{*}(C)^{D}\right), \quad G: \operatorname{eg}\left(\pi_{*}(C)^{D}\right) \rightarrow \operatorname{Tr}(k)
$$

defined (in the object part) as follows:

(a) If $M \in \operatorname{egl}(C)$ then $F(M)=\pi_{*}(M)^{D}$.

(b) If $X \in \operatorname{egr}\left(\pi_{*}(C)^{D}\right)$ then $G(X)=X^{\pi_{*}(C)^{D}}$.

We proved before (Theorem 3.15 and Lemma 3.9) that $F(M) \in$ $\operatorname{CoR}\left(\pi_{*}(C)^{D}\right)$ and that $(G \circ F)(M)=M^{C}$. The general theory of Grothendieck spectral sequences (see [2]) will give the theorem as soon as we prove

(i) $I$ injective in $\operatorname{Cor}(C)$ implies $F(I) G$-acyclic.

(ii) The derived functors of $F$ are $\pi_{*}$ composed with the $D$-cohomology functors.

It is enough to check (i) for $I=C$. In that case $F(I)=$ $\pi_{*}(C)^{D}$ and our conclusion follows from Lemma 2.1 .

As to (ii), since $\pi$ is normal, $\pi_{*}(C)$ is injective in C TR $(D)$. Consequently, the canonical resolution for $M$ as a $C$-comodule that we constructed in $\$ 2$, gives us (after applying $\pi_{*}$ ) an injective resolution $\pi_{*}(M)$ by injective $D$-comodules. Consequently the $i$ th derived functors of $F$ are functors $H^{\prime}\left(D, \pi_{*}()\right)$. 
In particular we can establish as corollaries the exactness of the Inflation Restriction sequence, as well as the exactness of the seven term sequence:

$$
\begin{aligned}
0 & \rightarrow H^{1}\left(\pi_{*}(C)^{D}, \pi_{*}(M)^{D}\right) \rightarrow H^{1}(C, M) \\
& \rightarrow H^{1}\left(D, \pi_{*}(M)\right)^{\pi_{*}(C)^{D}} \\
& \rightarrow H^{2}\left(\pi_{*}(C)^{D}, \pi_{*}(M)^{D}\right) \rightarrow \operatorname{Ker} \rho_{M} \\
& \rightarrow H^{1}\left(\pi_{*}(C)^{D}, H^{1}\left(D, \pi_{*}(M)\right)\right) \\
& \rightarrow H^{3}\left(\pi_{*}(C)^{D}, \pi_{*}(M)^{D}\right)
\end{aligned}
$$

$$
\text { where } \rho_{M}: H^{2}(C, M) \rightarrow H^{2}\left(D, \pi_{*}(M)\right)^{\pi_{*}(C)^{D}}
$$

is the so called restriction map.

It may be useful for some calculations to have a direct proof of the exactness of the sequence above. We shall sketch here such a proof.

Let $M$ be a $C$-comodule and consider an exact sequence in $\operatorname{CoR}(C)$

$$
0 \rightarrow M \rightarrow X \rightarrow L \rightarrow 0
$$

with $X$ coinduced. Taking the $D$ cohomology sequence of (1) we obtain an exact sequence and an isomorphism

$$
\begin{gathered}
0 \rightarrow M^{D} \rightarrow X^{D} \rightarrow L^{D} \rightarrow H^{1}(D, M) \rightarrow 0, \\
H^{1}(D, L) \cong H^{2}(D, M) .
\end{gathered}
$$

We have used here that $X$, when considered as a $D$-comodule, is injective. We split the sequence (2) into a pair of short exact sequences in the category $\operatorname{C} \pi\left(C^{D}\right)$ as follows:

$$
\begin{gathered}
0 \rightarrow M^{D} \rightarrow X^{D} \rightarrow Y \rightarrow 0, \\
0 \rightarrow Y \rightarrow L^{D} \rightarrow H^{1}(D, M) \rightarrow 0 .
\end{gathered}
$$

The long exact $C^{D}$ cohomology sequence that we obtain from (4) will give, after we identify all the terms, the seven term sequence.

$$
\begin{aligned}
0 & \rightarrow Y^{C^{D}} \rightarrow\left(L^{D}\right)^{C^{D}} \rightarrow H^{1}(D, M)^{C^{D}} \rightarrow H^{1}\left(C^{D}, Y\right) \\
& \rightarrow H^{1}\left(C^{D}, L^{D}\right) \rightarrow H^{1}\left(C^{D}, H^{1}(D, M)\right) \rightarrow H^{2}\left(C^{D}, Y\right) .
\end{aligned}
$$


Taking the $C^{D}$ cohomology sequence from (3) and the $C$ cohomology sequence from (1) we obtain:

$$
\begin{gathered}
0 \rightarrow M^{C} \rightarrow X^{C} \rightarrow Y^{C^{D}} \rightarrow H^{1}\left(C^{D}, M^{D}\right) \rightarrow 0, \\
H^{1}\left(C^{D}, Y\right) \cong H^{2}\left(C^{D}, M^{D}\right), H^{2}\left(C^{D}, Y\right) \cong H^{3}\left(C^{D}, M^{D}\right), \\
0 \rightarrow M^{C} \rightarrow X^{C} \rightarrow L^{C} \rightarrow H^{1}(C, M) \rightarrow 0, \\
H^{1}(C, L) \cong H^{2}(C, M) .
\end{gathered}
$$

It is easy to see that the map from $Y^{C^{D}}$ to $L^{C}$ in sequence (5) defines a map from $H^{1}\left(C^{D}, M^{D}\right)$ to $H^{1}(C, M)$ that is injective (see sequences (6) and (7)) and when substituted into (5) gives the seven term sequence

$$
\begin{aligned}
0 & \rightarrow H^{1}\left(C^{D}, M^{D}\right) \rightarrow H^{1}(C, M) \rightarrow H^{1}(D, M)^{C^{D}} \rightarrow H^{2}\left(C^{D}, M^{D}\right) \\
& \rightarrow H^{1}\left(C^{D}, L^{D}\right) \rightarrow H^{1}\left(C^{D}, H^{1}(D, M)\right) \rightarrow H^{3}\left(C^{D}, M^{D}\right) .
\end{aligned}
$$

Note that we also substituted the 4 th and 7 th term of (5) using the isomorphisms of $\left(6^{\prime}\right)$. The only term in $\left(5^{\prime}\right)$ that still needs identification is the 5 th. When we apply the first three terms of the sequence $\left(5^{\prime}\right)$ to $L$ we obtain the sequence:

$$
0 \rightarrow H^{1}\left(C^{D}, L^{D}\right) \rightarrow H^{1}(C, L) \rightarrow H^{1}(D, L) .
$$

Using $\left(2^{\prime}\right)$ and $\left(7^{\prime}\right)$ we have that $H^{1}\left(C^{D}, L^{D}\right) \cong \operatorname{Ker} \rho_{M}$, with $\rho_{M}: H^{2}(C, M) \rightarrow H^{2}(D, M)$.

Now consider Hopf Algebras $C$ and $D$ defined over a ring $R$, and let $\pi: C \rightarrow D$ be a surjective bialgebra map. Given any $D$-comodule $M$ we consider the $D$-comodule $\pi_{*}(C) \otimes M$ and the complex

$$
\begin{aligned}
E\left(\pi_{*}(C) \otimes M\right): 0 & \rightarrow \pi_{*}(C) \otimes M \stackrel{\delta_{1}}{\rightarrow} \pi_{*}(C) \otimes M \otimes D \rightarrow \cdots \\
& \rightarrow \pi_{*}(C) \otimes M \otimes D^{p-1} \stackrel{\delta_{p}}{\rightarrow} \pi_{*}(C) \otimes M \otimes D^{p} \rightarrow \cdots
\end{aligned}
$$

where the maps $\delta_{i}$ are defined inductively by formulas (3) and (4) of $\S 2$. We endow every object on the complex with a right $C$-comodule structure $\eta_{k}=\Delta \otimes \mathrm{id}_{M} \otimes \mathrm{id}_{D^{k}}$.

It is obvious that, with respect to these structures, the differentials of the complex are $C$-comodule maps. Let $\tilde{\eta}_{k}$ denote the left $C$-comodule structure constructed from $\eta_{k}$ in the same fashion as in the considerations before Lemma 4.8. The maps $\delta_{k}$ are now left $C$-comodule maps, and from the general considerations at the end of the proof of Theorem 3.6 
we deduce the existence of left $C$-comodule structures $\bar{\eta}_{k}$ on $H^{k}\left(D, \pi_{*}(C) \otimes M\right)$ that are compatible with the connecting homomorphisms and, at zero, coincide with the structure

$$
(\mathrm{id} \otimes \mathrm{id} \otimes \eta) s_{1,23}\left(\Delta \otimes \mathrm{id}_{M}\right) .
$$

Now let us look at the functor

$$
F_{\pi_{*}(C)}: \operatorname{CgR}(D) \rightarrow \operatorname{Cg}(C), \quad \text { where } F_{\pi_{*}(C)}(M)=\left(\pi_{*}(C) \otimes M\right)^{D} .
$$

$\left(\right.$ We endow $\left(\pi_{*}(C) \otimes M\right)^{D}$ with the structure (id $\left.\otimes \mathrm{id} \otimes \eta\right) s_{1,23}\left(\Delta \otimes \mathrm{id}_{M}\right)$ as we did in Lemma 4.8.) Consider $L$ : $\operatorname{C} \mathbb{R}(C) \rightarrow$ T $(R)$, where $L(M)=$ $M^{C}$.

Recall that in Lemma 4.8 we proved that $F_{\pi_{*}(C)}$ is naturally equivalent with the induced representation functor $\pi^{*}$ (see Definition 4.4).

LEMMA 5.2. The functor $L F_{\pi_{*}(C)}$ is naturally equivalent with the functor from $\operatorname{Col}(D)$ to $\pi(R)$ that sends $M$ to $M^{D}$.

Proof. We have noticed before that $F_{\pi_{*}(C)}$ is nothing else but $\pi^{*}$. Thus, our statement can be expressed in the following terms:

Let $M$ be a $D$-comodule and let $\phi_{M}: M \otimes C \rightarrow M \otimes D \otimes C$ be the map $\chi_{M} \otimes \mathrm{id}_{C}-\mathrm{id}_{M} \otimes(\pi \otimes \mathrm{id}) \Delta$. If we endow $M \otimes C$ with the structure id $\otimes \Delta$, then $\left(\operatorname{Ker} \phi_{M}\right)^{C} \cong M^{D}$. Consider the maps $E: M \otimes C \rightarrow M$, $E(m \otimes c)=\varepsilon(c) m$ and $\gamma: M \rightarrow M \otimes C, \gamma(m)=m \otimes 1$. Then we have

(a) $\gamma\left(M^{D}\right) \subset\left(\operatorname{Ker} \phi_{M}\right)^{C}$. This is because $\gamma(M) \subset(M \otimes C)^{C}$ (this is nothing but the assertion that $\Delta(1)=1 \otimes 1)$ and if $m \in M^{D}$ then $\chi m=$ $m \otimes 1$ and $\phi_{M} m=m \otimes 1 \otimes 1-m \otimes(\pi \otimes \mathrm{id}) \Delta(1)=m \otimes 1 \otimes 1-m \otimes$ $1 \otimes 1=0$.

(b) If $x \in(M \otimes C)^{C}$ then $x=E(x) \otimes 1$. This is because if $x=\sum m_{l} \otimes c_{l}$ and $\sum m_{l} \otimes \Delta c_{l}=\sum m_{l} \otimes c_{l} \otimes 1$, then applying id $\otimes \varepsilon \otimes \mathrm{id}$ yields $\sum m_{l} \otimes c_{i}=\sum \varepsilon\left(c_{l}\right) m_{i} \otimes 1$.

(c) If $x \in \operatorname{Ker} \phi_{M}$ then $\chi(E x)=($ id $\otimes \pi)(x)$. This is because if $x=$ $\sum m_{l} \otimes c_{l}$ and $\sum \chi m_{i} \otimes c_{l}=\sum m_{i} \otimes(\pi \otimes \mathrm{id}) \Delta c_{l}$, then applying id $\otimes \mathrm{id} \otimes \varepsilon$ yields $\sum \chi\left(m_{l} \varepsilon\left(c_{l}\right)\right)=\sum m_{l} \otimes \pi c_{l}$.

(d) $E \gamma=\mathrm{id}_{M}$.

Condition (b) tells us that if $x \in\left(\operatorname{Ker} \phi_{M}\right)^{C}$ then $x=\gamma E x$. This, together with (d), guarantees that $E$ restricted to $\left(\operatorname{Ker} \phi_{M}\right)^{C}$ and $\gamma$ restricted to $M^{D}$ are inverses to each other. Now from (b) and (c) we deduce that if $x \in\left(\operatorname{Ker} \phi_{M}\right)^{C}=\operatorname{Ker} \phi_{M} \cap(M \otimes C)^{C}$ then $\chi(E x)=($ id $\otimes \pi) x=$ $(\mathrm{id} \otimes \pi)(E x \otimes 1)=E x \otimes 1$. Thus $E\left(\left(\operatorname{Ker} \phi_{M}\right)^{C}\right) \subset M^{D}$. 
TheOrem 5.3. Let $C$ and $D$ be arbitrary Hopf Algebras defined over a ring $R$ that are flat as $R$-modules. Let $\pi$ be a surjective bialgebra map $\pi: C \rightarrow D$. Then, for every object $M$ in $\operatorname{COR}(D)$, there is a third quadrant spectral sequence, natural in $M,\left\{E_{r}^{p, q}\right\}$ such that

$$
E_{2}^{p, q}=H^{p}\left(C, H^{q}\left(D, \pi_{*}(C) \otimes M\right)\right) \underset{p}{\Rightarrow} H^{p+q}(D, M) .
$$

We regard $H^{q}\left(D, \pi_{*}(C) \otimes M\right)$ as a C-comodule with the structure $\bar{\eta}_{q}$ defined above.

Proof. The only part that still needs to be verified is that if $I$ is a relatively injective $D$-comodule, then $\pi^{*}(I)$ is $L$-acylic. It is enough to look at coinduced $D$-comodules of the form $X \otimes D$ with structure id ${ }_{X} \otimes \Delta$. In that case $\pi^{*}(X \otimes D) \cong X \otimes C$, as is seen from Lemma 4.5. By the very definition of our cohomology theory, $X \otimes C$ is $L$-acyclic.

In the case where $C$ is injective as a $D$-comodule, the spectral sequence collapses and gives us isomorphisms between $H^{p}\left(C, \pi^{*}(M)\right)$ and $H^{p}(D, M)$. The spectral sequence of Theorem 5.3 is the form that Shapiro's lemma takes in our cohomology theory.

\section{REFERENCES}

[1] E. Cline, B. Parshall and L. Scott, Induced modules and affine quotients, Math. Ann., 230 (1977), 1-14.

[2] A. Grothendieck, Sur quelques points d'Algebre Homologique, Tohoku Math. J., 9 (1957), 119-221.

[3] W. J. Haboush, Homogeneous vector bundles and reductive subgroups of reductive algebraic groups, Amer. J. Math., 100 No. 6, 1123-1137.

[4] G. Hochschild, Rationally injective modules for algebraic linear groups, Proc. Amer. Math. Soc., 14 No. 6, 880-883.

[5] Cohomology of algebraic linear groups, Illinois J. Math., 5 No. 3 (Sept. 1961), 492-519.

[6] M. E. Sweedler, Hopf Algebras, W. A. Benjamin, Inc., New York, 1969.

[7] M. Takeuchi, A correspondence between Hopf ideals and subHopf algebras, Manuscripta Math., 7 (1972), 251-270.

[8] Morita theorems for categories of comodules, J. Fac. Sci. Univ. Tokyo. Sect. IA Math., 24 No. 3 629-644.

Received March 27, 1981. Research partially supported by F.A.P.E.S.P. (São Paulo, Brasil).

Universidade De São Paulo

São Paulo, Brasil 



\section{PACIFIC JOURNAL OF MATHEMATICS}

EDITORS

Donald BABBITt (Managing Editor)

University of California

Los Angeles, CA 90024

Hugo Rossi

University of Utah

Salt Lake City, UT 84112

C. C. Moore and Arthur Ogus

University of California

Berkeley, CA 94720

\section{J. DugundiI}

Department of Mathematics

University of Southern California

Los Angeles, CA 90089-1113

R. Finn and H. SAMELSON

Stanford University

Stanford, CA 94305

\section{ASSOCIATE EDITORS}
R. ARENS
E. F. BECKENBACH
B. H. NEUMANN
F. WOLF
K. YOSHIDA (1906-1982)

\section{SUPPORTING INSTITUTIONS}

UNIVERSITY OF ARIZONA

UNIVERSITY OF BRITISH COLUMBIA

CALIFORNIA INSTITUTE OF TECHNOLOGY

UNIVERSITY OF CALIFORNIA

MONTANA STATE UNIVERSITY

UNIVERSITY OF NEVADA. RENO

NEW MEXICO STATE UNIVERSITY

OREGON STATE UNIVERSITY
UNIVERSITY OF OREGION

UNIVERSITY OF SOUTHERN CALIFORNIA

STANFORD UNIVERSITY

UNIVERSITY OF HAWAII

UNIVERSITY OF TOKYO

UNIVERSITY OF UTAH

WASHINGTON STATE UNIVERSITY

UNIVERSITY OF WASHINGTON 


\section{Pacific Journal of Mathematics}

Vol. 109, No. $1 \quad$ May, 1983

Donald George Babbitt and V. S. Varadarajan, Formal reduction theory of meromorphic differential equations: a group theoretic view $\ldots \ldots \ldots \ldots 1$

Jo-Ann Deborah Cohen, Norms on $F(X) \ldots \ldots \ldots \ldots \ldots \ldots \ldots \ldots \ldots \ldots$

Robert Fitzgerald, Witt kernels of function field extensions $\ldots \ldots \ldots \ldots \ldots 89$

Hervé Jacquet and Joseph Andrew Shalika, The Whittaker models of induced representations .............................. 107

Masakiti Kinukawa, Some generalizations of contraction theorems for

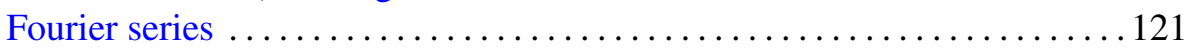

Joseph Weston Kitchen, Jr. and David A. Robbins, Sectional

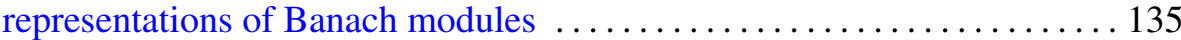

Victor Charles Pestien, Jr., Weak approximation of strategies in measurable gambling

Richard Scott Pierce and Charles Irvin Vinsonhaler, Realizing central

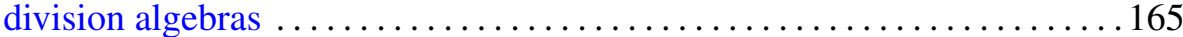

Walter Ricardo Ferrer Santos, Cohomology of comodules ............ 179

Marko Tadić, Harmonic analysis of spherical functions on reductive groups

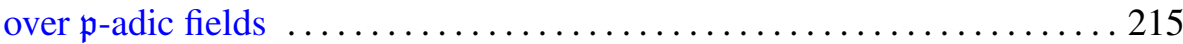

Lorenzo Traldi, The determinantal ideals of link modules. II . . . . . . . . 237

Alain J. Valette, A remark on the Kasparov groups $\operatorname{Ext}^{i}(A, B) \ldots \ldots \ldots 247$ 